\title{
Aerobic Oxidative Cyclization Under Pd(II) Catalysis: A Regioselective Approach to Heterocycles
}

\author{
Kai-Tai Yip, Jin-Heng Li, On-Yi Lee and Dan Yang* \\ Department of Chemistry, The University of Hong Kong, Pokfulam Road, Hong Kong, \\ P. R. China
}

\section{Supporting Information}

\section{General procedure:}

All reactions were performed in oven-dried flasks. Anhydrous ytterbium triflate and dichlorobis(acetonitrile)palladium(II) were purchased from Aldrich and Strem, respectively, and were used as received. Dichloroethane was distilled over calcium hydride under argon. 1,4-Dioxane (dried over molecular sieve) was purchased from Acros and used as received. THF was distilled from sodium metal-benzophenone ketyl before use. Thin-layer and flash column chromatography were performed on E. Merck silica gel 60 (230-400 mesh ASTM) using ethyl acetate/n-hexane as eluting solvents. Syringe pump addition was controlled by a Cole-Parmer ${ }^{\circledR}$ infusion dualsyringe pump.

${ }^{1} \mathrm{H}$ and ${ }^{13} \mathrm{C}$ NMR spectra were recorded in deuteriochloroform $\left(\mathrm{CDCl}_{3}\right)$ with tetramethylsilane (TMS) as internal standard at ambient temperature unless otherwise indicated on a Bruker Avance DPX 300 Fourier Transform Spectrometer operating at $300 \mathrm{MHz}$ for ${ }^{1} \mathrm{H}$ and $75 \mathrm{MHz}$ for ${ }^{13} \mathrm{C}$, or a Bruker Avance DPX 400 Fourier Transform Spectrometer operating at $400 \mathrm{MHz}$ for ${ }^{1} \mathrm{H}$ and $100 \mathrm{MHz}$ for ${ }^{13} \mathrm{C}$. Infrared absorption spectra were recorded as a solution in $\mathrm{CH}_{2} \mathrm{Cl}_{2}$ with a Bio-Rad FTS 165 Fourier Transform spectrophotometer. Mass spectra were recorded with a Finnigan MAT 95 mass spectrometer for both low resolution and high resolution mass spectra. 
Preparation of compounds 1a and 1c:

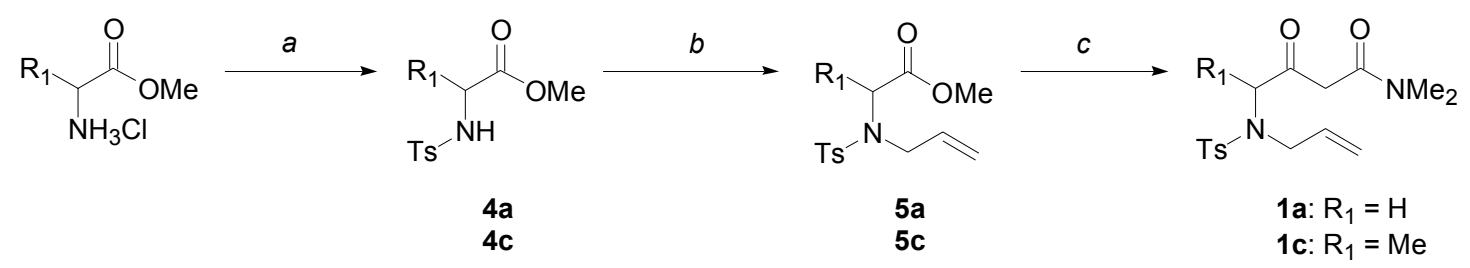

Reagents and conditions: (a) TsCl, $\mathrm{Et}_{3} \mathrm{~N}, \mathrm{CH}_{2} \mathrm{Cl}_{2}, 0{ }^{\circ} \mathrm{C}$; (b) Allyl bromide, $\mathrm{K}_{2} \mathrm{CO}_{3}$, acetone, reflux; (c) $N, N$-dimethylacetamide, LDA, THF, $-78{ }^{\circ} \mathrm{C}$.

\section{Typical procedure for the preparation of 4 :}

To a solution of glycine methyl ester hydrochloride (4.0 g, $31.9 \mathrm{mmol})$ in $\mathrm{CH}_{2} \mathrm{Cl}_{2}$ $(120 \mathrm{~mL})$ was added triethylamine $(9.8 \mathrm{~mL}, 70.1 \mathrm{mmol}) . p$-Toluenesulfonyl chloride (7.3 g, $38.2 \mathrm{mmol})$ in $\mathrm{CH}_{2} \mathrm{Cl}_{2}(40 \mathrm{~mL})$ was added slowly to the reaction mixture via addition funnel at $0{ }^{\circ} \mathrm{C}$. The reaction mixture was stirred at room temperature for $16 \mathrm{~h}$. The mixture was diluted with water $(60 \mathrm{~mL})$ and extracted with diethyl ether $(60 \mathrm{~mL}$ $\times 3)$. The combined organic layers were washed with brine $(60 \mathrm{~mL})$, dried over $\mathrm{MgSO}_{4}$ and concentrated in vacuo. The crude product was purified by flash column chromatography to give $4 \mathbf{a}^{1}$ (7.60 g, $\left.31.2 \mathrm{mmol}, 98 \%\right)$ as a white solid. ${ }^{1} \mathrm{H}$ NMR (400 $\left.\mathrm{MHz}, \mathrm{CDCl}_{3}\right) \delta 7.75(\mathrm{~d}, J=8.2 \mathrm{~Hz}, 2 \mathrm{H}), 7.31(\mathrm{~d}, J=8.1 \mathrm{~Hz}, 2 \mathrm{H}), 5.12$ (bs, 1H), 3.79 $(\mathrm{d}, J=5.5 \mathrm{~Hz}, 2 \mathrm{H}), 3.64(\mathrm{~s}, 3 \mathrm{H}), 2.42(\mathrm{~s}, 3 \mathrm{H}) ;{ }^{13} \mathrm{C} \mathrm{NMR}\left(75.5 \mathrm{MHz}, \mathrm{CDCl}_{3}\right) \delta$ 169.6, $144.2,136.5,130.1,127.6,52.9,44.4,21.9$.

4c: ${ }^{2}$ Yield 100\%; a white solid; ${ }^{1} \mathrm{H}$ NMR $\left(300 \mathrm{MHz}, \mathrm{CDCl}_{3}\right) \delta 7.72(\mathrm{~d}, J=8.2 \mathrm{~Hz}$, 2H), 7.27 (d, $J=8.1 \mathrm{~Hz}, 2 \mathrm{H}), 5.35$ (bs, 1H), 3.97 (q, $J=7.3 \mathrm{~Hz}, 1 \mathrm{H}), 3.52$ (s, 3H), $2.40(\mathrm{~s}, 3 \mathrm{H}), 1.36$ (d, $J=7.2 \mathrm{~Hz}, 3 \mathrm{H}) ;{ }^{13} \mathrm{C} \mathrm{NMR}\left(75.5 \mathrm{MHz}, \mathrm{CDCl}_{3}\right) \delta 172.9,143.9$, $137.1,129.9,127.5,52.8,51.7,21.8,20.0$. 


\section{Typical procedure for the preparation of 5:}

To a solution of $\mathbf{4 c}(4.0 \mathrm{~g}, 15.6 \mathrm{mmol})$ in acetone $(120 \mathrm{~mL})$ was added anhydrous potassium carbonate $(2.4 \mathrm{~g}, 17.1 \mathrm{mmol})$ and allyl bromide $(1.5 \mathrm{~mL}, 17.1 \mathrm{mmol})$. The reaction mixture was then stirred and refluxed for $14 \mathrm{~h}$. The mixture was diluted with water $(60 \mathrm{~mL})$ and acetone was evaporated. The residue was extracted with diethyl ether $(60 \mathrm{~mL} \times 3)$. The combined organic layers were dried over $\mathrm{MgSO}_{4}$ and concentrated in vacuo. The crude product was purified by flash column chromatography to give $\mathbf{5 c}^{3}$ (4.60 g, $\left.15.5 \mathrm{mmol}, 99 \%\right)$ as a colorless oil. ${ }^{1} \mathrm{H}$ NMR $\left(400 \mathrm{MHz} \mathrm{CDCl}_{3}\right) \delta 7.71(\mathrm{~d}, J=8.3 \mathrm{~Hz}, 2 \mathrm{H}), 7.29(\mathrm{~d}, J=8.1 \mathrm{~Hz}, 2 \mathrm{H}), 5.85-5.77$ (m, 1H), 5.21-5.08 (m, 2H), $4.65(\mathrm{q}, J=7.3 \mathrm{~Hz}, 1 \mathrm{H}), 3.95-3.81(\mathrm{~m}, 2 \mathrm{H}), 3.55(\mathrm{~s}, 3 \mathrm{H})$, $2.41(\mathrm{~s}, 3 \mathrm{H}), 1.40(\mathrm{~d}, J=7.3 \mathrm{~Hz}, 3 \mathrm{H}) ;{ }^{13} \mathrm{C} \mathrm{NMR}\left(100 \mathrm{MHz}, \mathrm{CDCl}_{3}\right) \delta 172.0,143.5$, $137.4,135.3,129.7,127.5,117.7,55.1,52.2,48.2,21.7,16.6$.

5a: Yield 98\%; a colorless oil; analytical TLC (silica gel 60), 20\% EtOAc in $n$-hexane, $\mathrm{R}_{f}=0.26 ;{ }^{1} \mathrm{H} \mathrm{NMR}\left(300 \mathrm{MHz}, \mathrm{CDCl}_{3}\right) \delta 7.71(\mathrm{~d}, J=8.3 \mathrm{~Hz}, 2 \mathrm{H}), 7.29(\mathrm{~d}, J=8.0 \mathrm{~Hz}$, 2H), 5.73-5.60 (m, 1H), $5.18(\mathrm{~s}, 1 \mathrm{H}), 5.15-5.12(\mathrm{dd}, J=7.3,1.1 \mathrm{~Hz}, 1 \mathrm{H}), 4.00(\mathrm{~s}$, $2 \mathrm{H}), 3.88(\mathrm{~d}, J=6.5 \mathrm{~Hz}, 2 \mathrm{H}), 3.61(\mathrm{~s}, 3 \mathrm{H}), 2.41(\mathrm{~s}, 3 \mathrm{H}) ;{ }^{13} \mathrm{C} \mathrm{NMR}\left(75.5 \mathrm{MHz}, \mathrm{CDCl}_{3}\right)$ $\delta 169.6,143.8,137.0,132.5,129.8,127.6,120.1,52.3,51.0,47.1,21.8 ; \mathrm{IR}\left(\mathrm{CH}_{2} \mathrm{Cl}_{2}\right)$ 3053, 2988, 2958, 1755, 1647, 1599, 1495, 1439, 1342, $1161 \mathrm{~cm}^{-1}$; LRMS (EI, $\left.20 \mathrm{eV}\right)$ $m / z 224\left(\mathrm{M}^{+}\right.$- COOMe, 50), 155 (Ts, 67), $128\left(\mathrm{M}^{+}\right.$- Ts, 100); HRMS (EI) for $\mathrm{C}_{13} \mathrm{H}_{17} \mathrm{O}_{4} \mathrm{NS}\left(\mathrm{M}^{+}\right)$: calcd 283.0878, found 283.0846. 


\section{Typical procedure for the preparation of 1:}

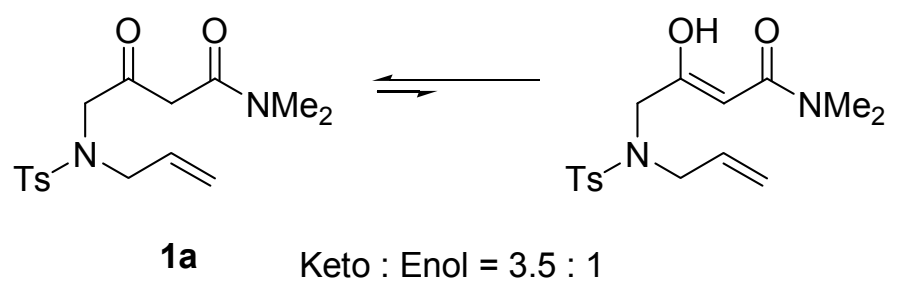

To a solution of LDA [freshly prepared from diisopropylamine $(0.91 \mathrm{~mL}, 6.5 \mathrm{mmol})$ and $n$-BuLi (2.45 $\mathrm{M}$ in hexane, $2.6 \mathrm{~mL}, 6.5 \mathrm{mmol})$ in $\mathrm{THF}(40 \mathrm{~mL})$ at $-78{ }^{\circ} \mathrm{C}$ ] was added $N, N$-dimethylacetamide $(0.55 \mathrm{~mL}, 5.9 \mathrm{mmol})$ at $-78{ }^{\circ} \mathrm{C}$. After $1 \mathrm{~h}$, a solution of $5 \mathbf{a}$ in THF $(20 \mathrm{~mL})$ was added slowly to the reaction mixture via a cannula. The mixture was stirred at $-78{ }^{\circ} \mathrm{C}$ for $1 \mathrm{~h}$ and warmed to room temperature slowly. After $14 \mathrm{~h}$, the reaction mixture was quenched with saturated $\mathrm{NH}_{4} \mathrm{Cl}$ solution $(60 \mathrm{~mL})$ and extracted with ethyl acetate $(60 \mathrm{~mL} \times 3)$. The combined organic layers were washed with brine $(60 \mathrm{~mL})$, dried over $\mathrm{MgSO}_{4}$ and concentrated in vacuo. The crude product was purified by flash column chromatography to give $1 \mathrm{a}(1.59 \mathrm{~g}, 4.7 \mathrm{mmol}, 80 \%)$ as a yellow syrup. Analytical TLC (silica gel 60), 80\% EtOAc in $n$-hexane, $\mathrm{R}_{f}=0.42 ;{ }^{1} \mathrm{H}$ NMR (400 MHz, $\left.\mathrm{CDCl}_{3}\right) \delta 14.87$ (bs, $0.22 \times 1 \mathrm{H}$, enol) 7.72-7.68 (m, 2H), 7.31-7.28 $(\mathrm{m}, 2 \mathrm{H}), 5.65-5.54(\mathrm{~m}, 1 \mathrm{H}), 5.36(\mathrm{~s}, 0.22 \times 1 \mathrm{H}, \mathrm{enol}), 5.16-5.11(\mathrm{~m}, 2 \mathrm{H}), 3.95(\mathrm{~s}$, $0.78 \times 2 \mathrm{H}$, keto), $3.86(\mathrm{~s}, 0.22 \times 2 \mathrm{H}$, enol $), 3.84(\mathrm{~d}, J=6.6 \mathrm{~Hz}, 0.22 \times 2 \mathrm{H}$, enol $), 3.80$ $(\mathrm{d}, J=6.7 \mathrm{~Hz}, 0.78 \times 2 \mathrm{H}$, keto), $3.66(\mathrm{~s}, 0.78 \times 2 \mathrm{H}$, keto), $3.00(\mathrm{~s}, 2 \mathrm{H}$, keto + enol), $2.95\left(\mathrm{~s}, 4 \mathrm{H}\right.$, keto + enol), $2.42(\mathrm{~s}, 0.78 \times 3 \mathrm{H}$, keto $), 2.41(\mathrm{~s}, 0.22 \times 3 \mathrm{H}$, enol $) ;{ }^{13} \mathrm{C}$ NMR $\left(100 \mathrm{MHz}, \mathrm{CDCl}_{3}\right) \delta 200.6,172.4,172.0,166.9,144.2,143.8,137.2,135.9$, $132.4,132.1,130.1,130.0,127.8,127.6,120.8,120.0,87.2$, 55.7, 52.2, 51.3, 49.3, 46.3, 38.2, 35.7, 29.9, 21.8; IR $\left(\mathrm{CH}_{2} \mathrm{Cl}_{2}\right)$ 3050, 2988, 2929, 1733, 1646, 1601, 1509 , 1427, 1341, $1160 \mathrm{~cm}^{-1}$; LRMS (EI, $\left.20 \mathrm{eV}\right) \mathrm{m} / \mathrm{z} 224\left(\mathrm{M}^{+}-(\mathrm{CO}) \mathrm{CH}_{2} \mathrm{CONMe}_{2}, 51\right)$, $183\left(\mathrm{M}^{+}-\mathrm{Ts}, 100\right), 155$ (Ts, 54), 114 (44); HRMS (EI) for $\mathrm{C}_{16} \mathrm{H}_{22} \mathrm{O}_{4} \mathrm{~N}_{2} \mathrm{~S}\left(\mathrm{M}^{+}\right)$: calcd 338.1300 , found 338.1306 . 


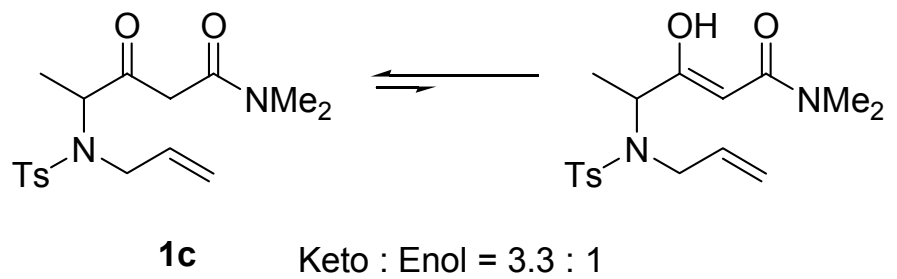

1c: Yield 50\%; an orange syrup; analytical TLC (silica gel 60), 80\% EtOAc in $n$ hexane, $\mathrm{R}_{f}=0.61 ;{ }^{1} \mathrm{H}$ NMR $\left(300 \mathrm{MHz}, \mathrm{CDCl}_{3}\right) \delta 15.11(\mathrm{~s}, 0.23 \times 1 \mathrm{H}$, enol) $7.74-7.70$ (m, 2H), 7.33-7.29 (m, 2H), 5.85-5.64 (m, 1H), $5.21(\mathrm{~s}, 0.23 \times 1 \mathrm{H}$, enol), 5.16-5.04 (m, 2H), 4.64-4.53 (m, 1H), $3.96(\mathrm{~d}, J=16.0 \mathrm{~Hz}, 0.77 \times 1 \mathrm{H}$, keto), 3.90-3.72 (m, $2 \mathrm{H}), 3.60(\mathrm{~d}, J=16.0 \mathrm{~Hz}, 0.77 \times 1 \mathrm{H}$, keto $), 2.98-2.41(\mathrm{~m}, 6 \mathrm{H}), 2.41(\mathrm{~s}, 0.77 \times 3 \mathrm{H}$, keto), 2.39 (s, $0.23 \times 3 \mathrm{H}$, enol), $1.33(\mathrm{~d}, J=7.1 \mathrm{~Hz}, 0.23 \times 3 \mathrm{H}$, enol), 1.12 (d, $J=7.0$ $\mathrm{Hz}, 0.77 \times 3 \mathrm{H}$, keto); ${ }^{13} \mathrm{C} \mathrm{NMR}\left(75.5 \mathrm{MHz}, \mathrm{CDCl}_{3}\right) \delta 202.4,174.8,171.9,167.5$, $144.0,143.4,138.0,137.2,136.1,133.8,130.1,129.7,127.6,127.5,119.7,117.3$, 87.1, 60.7, 56.2, 48.8, 47.8, 45.0, 37.9, 35.4, 21.7, 16.5, 12.7; IR $\left(\mathrm{CH}_{2} \mathrm{Cl}_{2}\right)$ 3051, 2988, 2940, 1729, 1644, 1596, 1503, 1341, $1161 \mathrm{~cm}^{-1}$; LRMS (EI, $\left.20 \mathrm{eV}\right) \mathrm{m} / z 239\left(\mathrm{M}^{+}\right.$(CO) $\left.\mathrm{CH}_{2} \mathrm{CONMe}_{2}, 14\right), 238$ (100), $197\left(\mathrm{M}^{+}\right.$- Ts, 20), 155 (Ts, 34), 114 (33); HRMS (EI) for $\mathrm{C}_{17} \mathrm{H}_{24} \mathrm{O}_{4} \mathrm{~N}_{2} \mathrm{~S}\left(\mathrm{M}^{+}\right)$: calcd 352.1457, found 352.1447.

\section{Preparation of compound $1 \mathrm{~b}$ :}

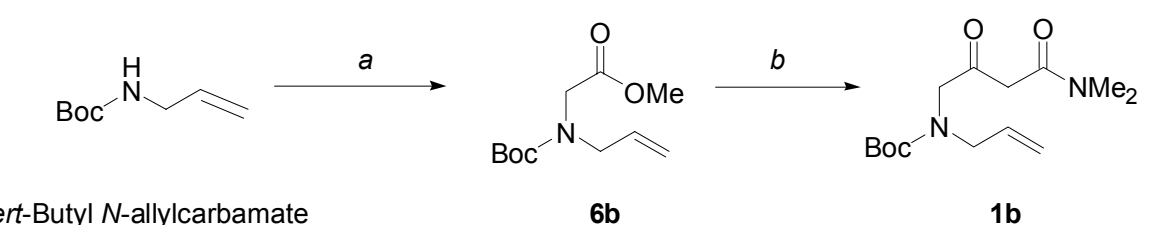

Reagents and conditions: (a) Methyl bromoacetate, $\mathrm{KH}, \mathrm{THF}, 0{ }^{\circ} \mathrm{C}$; (b) $\mathrm{N}, \mathrm{N}$ dimethylacetamide, LDA, THF, $-78^{\circ} \mathrm{C}$.

\section{Preparation of 6b:}

To a suspension of $\mathrm{KH}$ (30\% dispersion in oil, $4.1 \mathrm{~g}, 30.9 \mathrm{mmol})$ in THF $(100 \mathrm{~mL})$ was added a solution of tert-butyl $N$-allylcarbamate (4.4 g, $28.1 \mathrm{mmol})$ in THF (40 $\mathrm{mL}$ ) via a cannula at $0{ }^{\circ} \mathrm{C}$. After $30 \mathrm{~min}$, methyl bromoacetate $(2.8 \mathrm{~mL}, 30.9 \mathrm{mmol})$ was added slowly for $10 \mathrm{~min}$. The resulting solution was stirred at room temperature for $14 \mathrm{~h}$. The reaction mixture was quenched with saturated $\mathrm{NH}_{4} \mathrm{Cl}$ solution $(60 \mathrm{~mL})$ and the solvent was evaporated. The residue was extracted with diethyl ether $(60 \mathrm{~mL}$ 
$\times 3$ ). The combined organic layers were dried over $\mathrm{MgSO}_{4}$ and concentrated in vacuo. The crude product was purified by flash column chromatography to give $6 \mathbf{b}$ (4.00 g, $17.4 \mathrm{mmol}, 62 \%$ ) as a yellow oil. Analytical TLC (silica gel 60), 20\% EtOAc in $n$ hexane, $\mathrm{R}_{f}=0.22 ;{ }^{1} \mathrm{H}$ NMR $\left(400 \mathrm{MHz}, \mathrm{CDCl}_{3}\right) \delta 5.82-5.74(\mathrm{~m}, 1 \mathrm{H}), 5.18-5.10(\mathrm{~m}$, 2H), 3.96 (s, 2H), 3.89 (d, $J=5.5 \mathrm{~Hz}, 1 \mathrm{H}), 3.84$ (s, 1H), 3.73 (s, 3H), 1.47 (s, 5H, major conformer), 1.43 (s, $4 \mathrm{H}$, minor conformer); $\left.{ }^{13} \mathrm{C} \mathrm{NMR} \mathrm{(75.5} \mathrm{MHz}, \mathrm{CDCl}_{3}\right) \delta$ 170.8, 155.9, 155.4, 134.0, 133.9, 117.9, 117.1, 80.7, 52.8, 52.2, 51.0, 50.6, 48.2, 47.8, 28.6, 28.5; IR $\left(\mathrm{CH}_{2} \mathrm{Cl}_{2}\right)$ 3062, 3053, 2988, 2952, 1752, 1701, 1642, 1456, $1169 \mathrm{~cm}^{-1}$; LRMS (EI, $20 \mathrm{eV}) m / z 203\left(\mathrm{M}^{+}-\mathrm{CH}=\mathrm{CH}_{2}, 16\right), 199$ (69), 185 (100), $171\left(\mathrm{M}^{+}\right.$$\left.\mathrm{C}\left(\mathrm{CH}_{3}\right)_{3}, 60\right)$; HRMS (EI) for $\mathrm{C}_{11} \mathrm{H}_{19} \mathrm{O}_{4} \mathrm{~N}\left(\mathrm{M}^{+}\right)$: calcd 229.1314, found 229.1300.

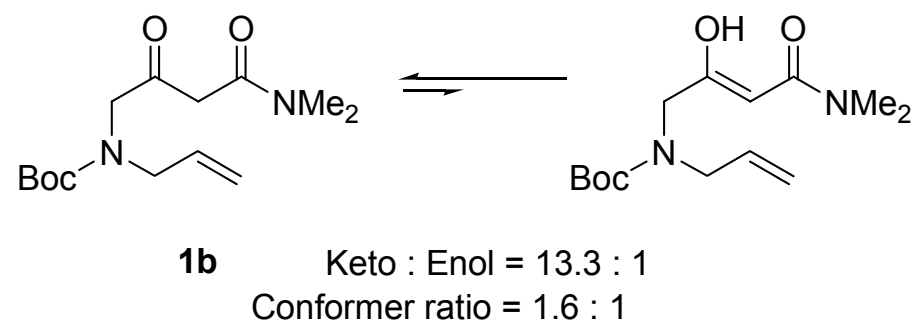

1b: Yield 40\%; a yellow oil; analytical TLC (silica gel 60), 80\% EtOAc in $n$-hexane, $\mathrm{R}_{f}=0.39 ;{ }^{1} \mathrm{H}$ NMR $\left(300 \mathrm{MHz}, \mathrm{CDCl}_{3}\right) \delta 14.93(\mathrm{~s}, 0.07 \times 1 \mathrm{H}$, enol $), 5.83-5.70(\mathrm{~m}$, $1 \mathrm{H}), 5.15-5.11(\mathrm{~m}, 2 \mathrm{H}), 4.07(\mathrm{~s}, 2 \mathrm{H}), 3.92-3.86(\mathrm{~m}, 2 \mathrm{H}), 3.56(\mathrm{~s}, 0.62 \times 2 \mathrm{H}$, major conformer), $3.52(\mathrm{~s}, 0.38 \times 2 \mathrm{H}$, minor conformer), 3.01-2.97 (m, 6H), $1.46(\mathrm{~s}, 0.62 \times$ 9H, major conformer), 1.42 (s, $0.38 \times 9 \mathrm{H}$, minor conformer); ${ }^{13} \mathrm{C} \mathrm{NMR}(75.5 \mathrm{MHz}$, $\left.\mathrm{CDCl}_{3}\right) \delta 201.1,200.6,174.2,172.2,167.0,166.4,156.0,155.3,133.8,133.6,117.8$, $117.1,86.0,85.0,80.7,80.4,56.2,51.4,50.7,49.1,46.5,46.3,38.1,35.7,28.5$; IR $\left(\mathrm{CH}_{2} \mathrm{Cl}_{2}\right)$ 3051, 2988, 2929, 1744, 1693, 1647, $1464 \mathrm{~cm}^{-1}$; LRMS (EI, $\left.20 \mathrm{eV}\right) \mathrm{m} / z 284$ $\left(\mathrm{M}^{+}, 17\right), 228$ (15), 211 (28), 184 (18), 129 (11), 114 (100); HRMS (EI) for $\mathrm{C}_{14} \mathrm{H}_{24} \mathrm{O}_{4} \mathrm{~N}_{2}\left(\mathrm{M}^{+}\right)$: calcd 284.1736, found 284.1729. 
Preparation of compounds 1d and 1e:

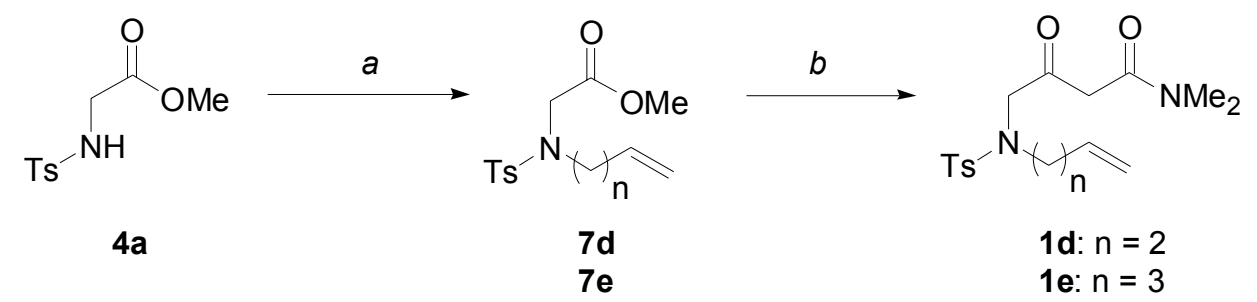

Reagents and conditions: (a) $\mathrm{K}_{2} \mathrm{CO}_{3}, \mathrm{KI}, \mathrm{Br}{ }_{\mathrm{n}}$, Acetone, reflux; (b) $N, N$ dimethylacetamide, LDA, THF, $-78^{\circ} \mathrm{C}$.

\section{Typical procedure for the preparation of 7:}

To a solution of $4 \mathbf{a}(2.0 \mathrm{~g}, 8.22 \mathrm{mmol})$ in acetone $(40 \mathrm{~mL})$ was added anhydrous potassium carbonate (1.25 g, $9.04 \mathrm{mmol}$ ), anhydrous potassium iodide (682 mg, 4.11 mmol) and 5-bromo-1-pentene $(1.1 \mathrm{~mL}, 9.04 \mathrm{mmol})$. The reaction mixture was stirred and refluxed for $18 \mathrm{~h}$. The mixture was diluted with water $(40 \mathrm{~mL})$ and acetone was evaporated. The residue was extracted with diethyl ether $(40 \mathrm{~mL} \times 3)$. The combined organic layers were dried over $\mathrm{MgSO}_{4}$ and concentrated in vacuo. The crude product was purified by flash column chromatography to give 7 e $(2.06 \mathrm{~g}, 6.62 \mathrm{mmol}, 81 \%)$ as a yellow oil. Analytical TLC (silica gel 60), 20\% EtOAc in $n$-hexane, $\mathrm{R}_{f}=0.40 ;{ }^{1} \mathrm{H}$ NMR (400 MHz, $\left.\mathrm{CDCl}_{3}\right) \delta 7.71(\mathrm{~d}, J=8.2 \mathrm{~Hz}, 2 \mathrm{H}), 7.29(\mathrm{~d}, J=8.3 \mathrm{~Hz}, 2 \mathrm{H})$, 5.78-5.69 (m, 1H), $4.99(\mathrm{dd}, J=15.5,1.2 \mathrm{~Hz}, 1 \mathrm{H}), 4.96(\mathrm{dd}, J=9.8,1.2 \mathrm{~Hz}, 1 \mathrm{H})$, $4.04(\mathrm{~s}, 2 \mathrm{H}), 3.63(\mathrm{~s}, 3 \mathrm{H}), 3.23(\mathrm{t}, J=7.5 \mathrm{~Hz}, 2 \mathrm{H}), 2.42(\mathrm{~s}, 3 \mathrm{H}), 2.03(\mathrm{q}, J=7.0 \mathrm{~Hz}$, $2 \mathrm{H}$ ), 1.63 (quintet, $\left.J=7.4 \mathrm{~Hz}, 2 \mathrm{H}) ;{ }^{13} \mathrm{C} \mathrm{NMR} \mathrm{(100} \mathrm{MHz,} \mathrm{CDCl}_{3}\right) \delta$ 169.4, 143.4, 137.3, 136.7, 129.5, 127.3, 115.3, 52.1, 48.0, 30.5, 27.0, 21.5; IR $\left(\mathrm{CH}_{2} \mathrm{Cl}_{2}\right)$ 3063, 3048, 2989, 2957, 1756, 1648, 1603, 1495, 1437, 1341, $1160 \mathrm{~cm}^{-1}$; LRMS (EI, $20 \mathrm{eV}$ ) $m / z 256\left(\mathrm{M}^{+}-\mathrm{CH}_{2} \mathrm{CH}_{2} \mathrm{CH}=\mathrm{CH}_{2}, 72\right), 252$ (65), 198 (39), 155 (100); HRMS (EI) for $\mathrm{C}_{15} \mathrm{H}_{21} \mathrm{O}_{4} \mathrm{NS}\left(\mathrm{M}^{+}\right)$: calcd 311.1191, found 311.1185.

7d: Yield 72\% (based on 53\% conversion) as a yellow oil; analytical TLC (silica gel 60), 20\% EtOAc in $n$-hexane, $\mathrm{R}_{f}=0.40 ;{ }^{1} \mathrm{H}$ NMR $\left(400 \mathrm{MHz}, \mathrm{CDCl}_{3}\right) \delta 7.72(\mathrm{~d}, J=$ $8.3 \mathrm{~Hz}, 2 \mathrm{H}), 7.30(\mathrm{~d}, J=8.0 \mathrm{~Hz}, 2 \mathrm{H}), 5.73-5.66(\mathrm{~m}, 1 \mathrm{H}), 5.06(\mathrm{dd}, J=15.6,1.4 \mathrm{~Hz}$, $1 \mathrm{H}), 5.03(\mathrm{dd}, J=8.9,1.4 \mathrm{~Hz}, 1 \mathrm{H}), 4.07(\mathrm{~s}, 2 \mathrm{H}), 3.63(\mathrm{~s}, 3 \mathrm{H}), 3.31(\mathrm{t}, J=6.2 \mathrm{~Hz}, 2 \mathrm{H})$, $2.42(\mathrm{~s}, 3 \mathrm{H}), 2.29$ (q, $J=6.1 \mathrm{~Hz}, 2 \mathrm{H}) ;{ }^{13} \mathrm{C} \mathrm{NMR}\left(100 \mathrm{MHz}, \mathrm{CDCl}_{3}\right) \delta 169.4,143.4$, 
136.8, 134.4, 129.5, 127.4, 117.3, 52.1, 48.1, 47.7, 32.6, 21.5; IR $\left(\mathrm{CH}_{2} \mathrm{Cl}_{2}\right)$ 3074, 3050, 2956, 1755, 1642, 1600, 1495, 1439, 1343, $1160 \mathrm{~cm}^{-1}$; LRMS (EI, $\left.20 \mathrm{eV}\right) \mathrm{m} / \mathrm{z}$ $256\left(\mathrm{M}^{+}-\mathrm{CH}_{2} \mathrm{CH}=\mathrm{CH}_{2}, 100\right), 228$ (11), 155 (73); HRMS (EI) for $\mathrm{C}_{14} \mathrm{H}_{19} \mathrm{O}_{4} \mathrm{NS}\left(\mathrm{M}^{+}\right)$: calcd 297.1035, found 297.1023.

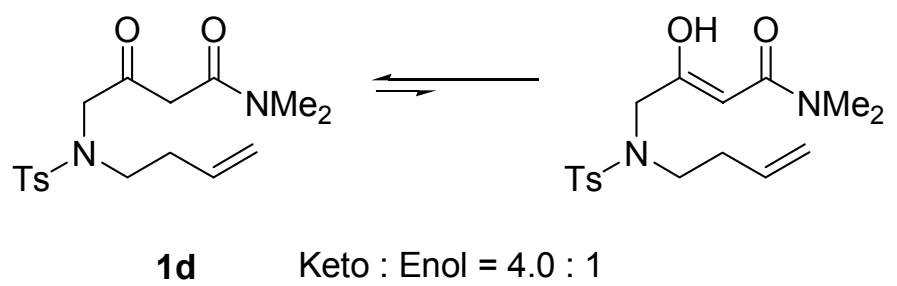

1d: Yield 65\%; an orange syrup; analytical TLC (silica gel 60), 100\% EtOAc, $\mathrm{R}_{f}=$ 0.47; ${ }^{1} \mathrm{H}$ NMR (400 MHz, $\left.\mathrm{CDCl}_{3}\right) \delta 14.98(\mathrm{~s}, 0.20 \times 1 \mathrm{H}$, enol), 7.73-7.68 (m, 2H), 7.32-7.29 (m, 2H), 5.73-5.63 (m, 1H), 5.36 (s, $0.20 \times 1 \mathrm{H}$, enol), 5.07-5.00 (m, 2H), $4.05(\mathrm{~s}, 0.80 \times 2 \mathrm{H}$, keto $), 3.90(\mathrm{~s}, 0.20 \times 2 \mathrm{H}$, enol $), 3.67(\mathrm{~s}, 0.80 \times 2 \mathrm{H}$, keto $)$, 3.28-3.21 (m, 2H), $3.01(\mathrm{~s}, 2 \mathrm{H}$, keto + enol), $2.96(\mathrm{~s}, 4 \mathrm{H}$, keto + enol), $2.43(\mathrm{~s}, 0.80 \times$ $3 \mathrm{H}$, keto), $2.42\left(\mathrm{~s}, 0.20 \times 3 \mathrm{H}\right.$, enol), $2.31-2.23(\mathrm{~m}, 2 \mathrm{H}) ;{ }^{13} \mathrm{C} \mathrm{NMR}\left(100 \mathrm{MHz}, \mathrm{CDCl}_{3}\right)$ $\delta 200.6,172.5,171.9,167.0,144.0,143.7,136.8,135.8,134.6,129.9,127.7,127.5$, $117.4,87.2,57.1,50.7,49.1,48.6,45.7,38.0,35.6,32.8,32.5,21.7 ; \mathrm{IR}\left(\mathrm{CH}_{2} \mathrm{Cl}_{2}\right)$ 3051, 2988, 2928, 1720, 1646, 1598, 1496, 1450, 1340, $1161 \mathrm{~cm}^{-1}$; LRMS (EI, $20 \mathrm{eV}$ ) $m / z 312\left(\mathrm{M}^{+}-\mathrm{CH}_{2} \mathrm{CH}=\mathrm{CH}_{2}, 14\right), 238$ (25), 198 (100), 184 (27), 155 (48); HRMS (EI) for $\mathrm{C}_{17} \mathrm{H}_{24} \mathrm{O}_{4} \mathrm{~N}_{2} \mathrm{~S}\left(\mathrm{M}^{+}\right)$: calcd 352.1457, found 352.1454 .

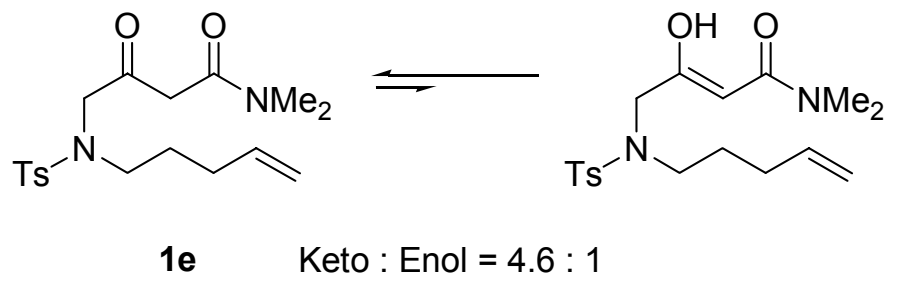

1e: Yield 69\%; an orange syrup; analytical TLC (silica gel 60), 80\% EtOAc in $n$ hexane, $\mathrm{R}_{f}=0.38 ;{ }^{1} \mathrm{H} \mathrm{NMR}\left(400 \mathrm{MHz}, \mathrm{CDCl}_{3}\right) \delta 14.94(\mathrm{~s}, 0.18 \times 1 \mathrm{H}$, enol), 7.72-7.68 (m, 2H), 7.33-7.31 (m, 2H), 5.78-5.68 (m, 1H), $5.39(\mathrm{~s}, 0.18 \times 1 \mathrm{H}$, enol), 5.02-4.95 (m, 2H), $3.98(\mathrm{~s}, 0.82 \times 2 \mathrm{H}$, keto), $3.87(\mathrm{~s}, 0.18 \times 2 \mathrm{H}$, enol), 3.70 (s, $0.82 \times$ 2H, keto), 3.20-3.10 (m, 2H), 3.03 (s, 2H, keto + enol), 2.97 (s, 4H, keto + enol), 2.43 (s, $0.82 \times 3 \mathrm{H}$, keto), $2.42(\mathrm{~s}, 0.18 \times 3 \mathrm{H}$, enol), 2.05-2.01 (m, 2H), 1.65-1.57 (m, 2H); ${ }^{13} \mathrm{C}$ NMR $\left(100 \mathrm{MHz}, \mathrm{CDCl}_{3}\right) \delta 200.8,172.8,172.1,167.1,144.1,143.7,137.6,137.5$, 
$137.0,136.7,130.1,130.0,127.9,127.6,115.8,115.7,87.3,57.2,50.9,49.7,49.1$, 45.9, 38.2, 35.7, 31.1, 30.9, 30.0, 27.6, 27.4, 21.8; IR $\left(\mathrm{CH}_{2} \mathrm{Cl}_{2}\right)$ 3058, 2990, 2940, 1729, 1655, 1596, 1493, $1326 \mathrm{~cm}^{-1}$; LRMS (EI, $\left.20 \mathrm{eV}\right) \mathrm{m} / z 252\left(\mathrm{M}^{+}-\right.$ (CO) $\left.\mathrm{CH}_{2} \mathrm{CONMe}_{2}, 36\right), 211\left(\mathrm{M}^{+}\right.$- Ts, 100), 198 (27); HRMS (EI) for $\mathrm{C}_{18} \mathrm{H}_{26} \mathrm{O}_{4} \mathrm{~N}_{2} \mathrm{~S}$ $\left(\mathrm{M}^{+}\right)$: calcd 366.1613, found 366.1607.

\section{Preparation of compounds $1 \mathrm{f}$ and $1 \mathrm{~h}$ :}

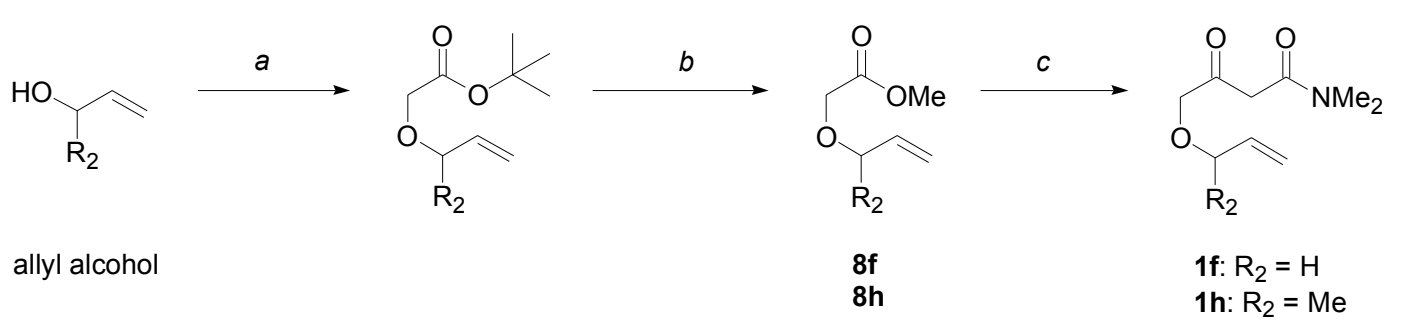

Reagents and conditions: (a) tert-Butyl bromoacetate, 50\% aqueous $\mathrm{NaOH},(n$ $\mathrm{Bu})_{4} \mathrm{NHSO}_{4}$, benzene, $0{ }^{\circ} \mathrm{C}$; (b) $\mathrm{SOCl}_{2}, \mathrm{MeOH}, 0{ }^{\circ} \mathrm{C}$; (c) $N, N$-dimethylacetamide, LDA, THF, $-78{ }^{\circ} \mathrm{C}$.

\section{Typical procedure for the preparation of 8:}

To a solution of allyl alcohol $(3.0 \mathrm{~g}, 51.6 \mathrm{mmol})$ in benzene $(60 \mathrm{~mL})$ was added tetrabutylammonium hydrogen sulfate $(1.8 \mathrm{~g}, 5.2 \mathrm{mmol})$ and $50 \%$ aqueous $\mathrm{NaOH}$ solution $(60 \mathrm{~mL})$ at $0{ }^{\circ} \mathrm{C}$. With vigorous stirring, tert-butyl bromoacetate $(11.4 \mathrm{~mL}$, $77.5 \mathrm{mmol}$ ) was added slowly and the mixture was warmed to room temperature. After $16 \mathrm{~h}$, the mixture was diluted with $n$-pentane $(60 \mathrm{~mL})$ and water $(60 \mathrm{~mL})$. The organic layer was separated and the aqueous layer was extracted with diethyl ether $(60 \mathrm{~mL} \times 3)$. The combined organic layers were concentrated in vacuo. The crude product was dissolved in methanol $(170 \mathrm{~mL})$. With vigorous stirring at $0{ }^{\circ} \mathrm{C}$, thionyl chloride $(11.2 \mathrm{~mL}, 155 \mathrm{mmol})$ was added via addition funnel for $10 \mathrm{~min}$. After $12 \mathrm{~h}$, the reaction mixture was concentrated in vacuo. The residue was purified by flash column chromatography ( $n$-pentane/ $\mathrm{Et}_{2} \mathrm{O}$ as solvents) to give $\mathbf{8 \mathbf { f } ^ { 4 }}$ (5.00 g, $38.4 \mathrm{mmol}$, $74 \%$ over 2 steps) as a yellow oil. ${ }^{1} \mathrm{H}$ NMR $\left(400 \mathrm{MHz}, \mathrm{CDCl}_{3}\right) \delta 5.89-5.82(\mathrm{~m}, 1 \mathrm{H})$, $5.25(\mathrm{~d}, J=17.2 \mathrm{~Hz}, 1 \mathrm{H}), 5.17(\mathrm{~d}, J=10.3 \mathrm{~Hz}, 1 \mathrm{H}), 4.04(\mathrm{~s}, 4 \mathrm{H}), 3.70(\mathrm{~s}, 3 \mathrm{H}) ;{ }^{13} \mathrm{C}$ NMR (75.5 MHz, $\left.\mathrm{CDCl}_{3}\right) \delta 170.9,133.9,118.3,72.5,67.2,51.9$. 
$\mathbf{8 h}^{5}$ : Yield 87\% (over 2 steps); a yellow oil; ${ }^{1} \mathrm{H}$ NMR (300 MHz, $\left.\mathrm{CDCl}_{3}\right) \delta 5.77-5.65$ (m, 1H), 5.19 (d, $J=17.6 \mathrm{~Hz}, 1 \mathrm{H}), 5.17$ (d, $J=10.0 \mathrm{~Hz}, 1 \mathrm{H}), 4.09$ (d, $J=16.4 \mathrm{~Hz}$, $1 \mathrm{H}), 4.00(\mathrm{~d}, J=16.4 \mathrm{~Hz}, 1 \mathrm{H}), 3.93$ (quintet, $J=6.9 \mathrm{~Hz}, 1 \mathrm{H}), 3.74(\mathrm{~s}, 3 \mathrm{H}), 1.31$ (d, $J$ $=6.4 \mathrm{~Hz}, 3 \mathrm{H}) ;{ }^{13} \mathrm{C} \mathrm{NMR}\left(75.5 \mathrm{MHz}, \mathrm{CDCl}_{3}\right) \delta 171.4,139.3,117.5,78.2,65.7,52.0$, 21.4 .

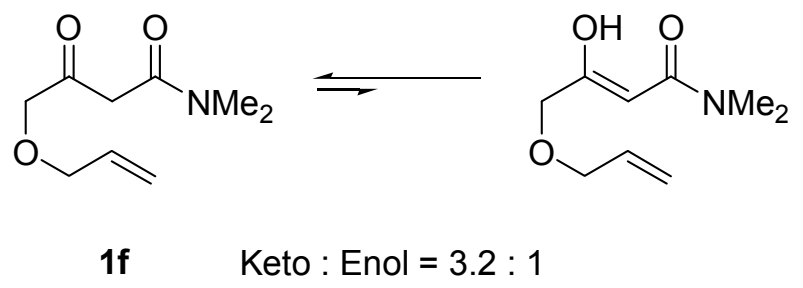

1f: Yield 66\%; a yellow oil; analytical TLC (silica gel 60), 60\% EtOAc in $n$-hexane, $\mathrm{R}_{f}=0.20 ;{ }^{1} \mathrm{H} \mathrm{NMR}\left(300 \mathrm{MHz}, \mathrm{CDCl}_{3}\right) \delta 14.86(\mathrm{~s}, 0.24 \times 1 \mathrm{H}$, enol), 5.97-5.84 (m, $1 \mathrm{H}), 5.46(\mathrm{~s}, 0.24 \times 1 \mathrm{H}$, enol), 5.33-5.19 (m, 2H), $4.17(\mathrm{~s}, 0.76 \times 2 \mathrm{H}$, keto), 4.07 (d, $J$ $=5.6 \mathrm{~Hz}, 2 \mathrm{H}), 4.03(\mathrm{~s}, 0.24 \times 2 \mathrm{H}, \mathrm{enol}), 3.60(\mathrm{~s}, 0.76 \times 2 \mathrm{H}$, keto $), 3.01(\mathrm{~s}, 4 \mathrm{H}$, keto + enol), 2.97 (s, 2H, keto + enol); $\left.{ }^{13} \mathrm{C} \mathrm{NMR} \mathrm{(75.5} \mathrm{MHz,} \mathrm{CDCl}_{3}\right) \delta$ 203.0, 173.6, 172.1, $166.8,134.2,133.9,118.0,117.6,85.5,75.0,72.5,72.1,69.3,45.4,38.0,35.4$; IR $\left(\mathrm{CH}_{2} \mathrm{Cl}_{2}\right)$ 3051, 2996, 2934, 1730, 1646, $1596 \mathrm{~cm}^{-1}$; LRMS (EI, $\left.20 \mathrm{eV}\right) \mathrm{m} / z 144\left(\mathrm{M}^{+}\right.$ - $\mathrm{CH}_{2} \mathrm{CH}=\mathrm{CH}_{2}, 66$ ), 129 (66), 127 (11), 114 (100); HRMS (EI) for $\mathrm{C}_{9} \mathrm{H}_{15} \mathrm{O}_{3} \mathrm{~N}\left(\mathrm{M}^{+}\right.$): calcd 185.1052, found 185.1050 .

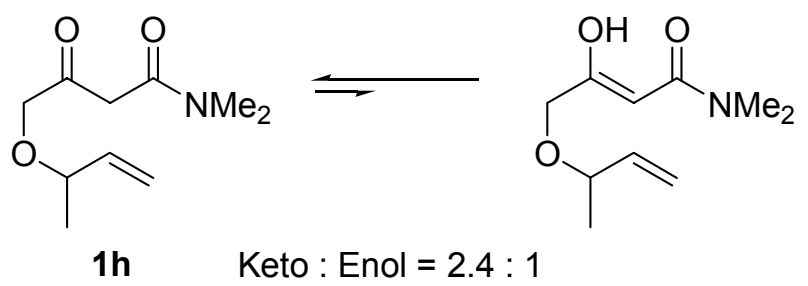

1h: Yield 58\%; a yellow oil; analytical TLC (silica gel 60), 60\% EtOAc in $n$-hexane, $\mathrm{R}_{f}=0.29 ;{ }^{1} \mathrm{H} \mathrm{NMR}\left(300 \mathrm{MHz}, \mathrm{CDCl}_{3}\right) \delta 14.85(\mathrm{~s}, 0.29 \times 1 \mathrm{H}$, enol), 5.80-5.65 (m, $1 \mathrm{H}), 5.46(\mathrm{~s}, 0.29 \times 1 \mathrm{H}, \mathrm{enol}), 5.24-5.16(\mathrm{~m}, 2 \mathrm{H}), 4.16(\mathrm{~d}, J=17.2 \mathrm{~Hz}, 1 \mathrm{H}), 4.07$ (d, $J=17.3 \mathrm{~Hz}, 1 \mathrm{H}), 3.96-3.85(\mathrm{~m}, 1 \mathrm{H}), 3.64(\mathrm{~d}, J=15.5 \mathrm{~Hz}, 0.71 \times 1 \mathrm{H}, \mathrm{keto}), 3.57(\mathrm{~d}$, $J=15.5 \mathrm{~Hz}, 0.71 \times 1 \mathrm{H}$, keto), $3.01(\mathrm{~s}, 4 \mathrm{H}$, keto + enol), $2.97(\mathrm{~s}, 2 \mathrm{H}$, keto + enol), 1.29 $(\mathrm{d}, J=6.4 \mathrm{~Hz}, 3 \mathrm{H}) ;{ }^{13} \mathrm{C} \mathrm{NMR}\left(75.5 \mathrm{MHz}, \mathrm{CDCl}_{3}\right) \delta 203.6,174.3,172.3,166.9,139.6$, $139.2,117.3,116.8,85.5,78.2,77.6,73.5,67.6,45.8,38.2,35.6,21.4,21.3$; IR $\left(\mathrm{CH}_{2} \mathrm{Cl}_{2}\right)$ 3046, 2985, 2934, 1729, 1646, $1596 \mathrm{~cm}^{-1}$; LRMS (EI, $\left.20 \mathrm{eV}\right) \mathrm{m} / \mathrm{z} 199\left(\mathrm{M}^{+}\right.$, 
12), 144 (61), 129 (45), 115 (100); HRMS (EI) for $\mathrm{C}_{10} \mathrm{H}_{17} \mathrm{O}_{3} \mathrm{~N}\left(\mathrm{M}^{+}\right)$: calcd 199.1208, found 199.1198 .

\section{Preparation of compound 1g:}

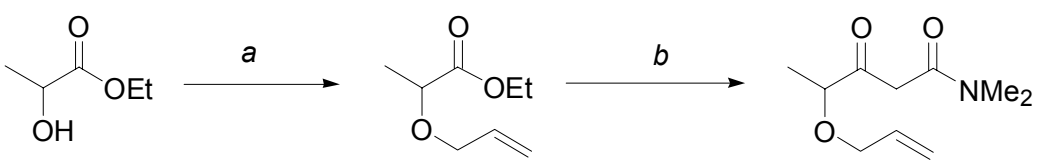

Ethyl lactate

$9 g$

19

Reagents and conditions: (a) Allyl bromide, $\mathrm{Ag}_{2} \mathrm{O}, \mathrm{Et}_{2} \mathrm{O}, \mathrm{rt}$; (b) $\mathrm{N}, \mathrm{N}$ dimethylacetamide, LDA, THF, $-78^{\circ} \mathrm{C}$.

For the preparation of $9 \mathrm{~g}$, see reference 6 .

9g ${ }^{6}$ : Yield 91\%; a yellow oil; ${ }^{1} \mathrm{H}$ NMR (300 MHz, $\left.\mathrm{CDCl}_{3}\right) \delta 5.99-5.86(\mathrm{~m}, 1 \mathrm{H}), 5.29$ $(\mathrm{dd}, J=17.2,1.6 \mathrm{~Hz}, 1 \mathrm{H}), 5.20(\mathrm{dd}, J=10.4,1.4 \mathrm{~Hz}, 1 \mathrm{H}), 4.22(\mathrm{q}, J=7.2 \mathrm{~Hz}, 2 \mathrm{H})$, $4.15(\mathrm{dd}, J=12.6,5.5 \mathrm{~Hz}, 1 \mathrm{H}), 4.00(\mathrm{q}, J=6.8 \mathrm{~Hz}, 1 \mathrm{H}), 3.94$, (dd, $J=12.5,6.1 \mathrm{~Hz}$, $1 \mathrm{H}), 1.42(\mathrm{~d}, J=6.9 \mathrm{~Hz}, 3 \mathrm{H}) ;{ }^{13} \mathrm{C} \mathrm{NMR}\left(75.5 \mathrm{MHz}, \mathrm{CDCl}_{3}\right) \delta 173.5,134.5,117.8$, $74.3,71.3,61.0,18.9,14.5$.

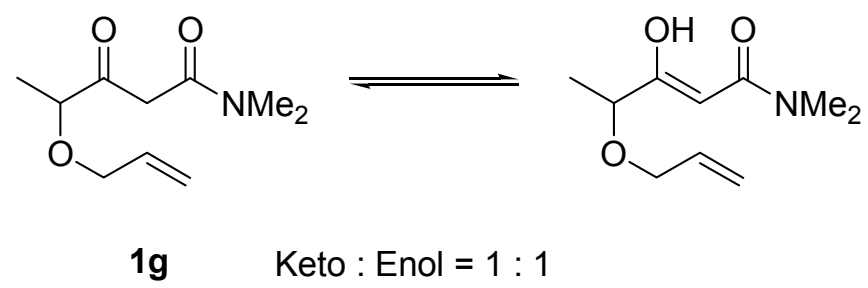

1g: Yield 48\%; a yellow oil; analytical TLC (silica gel 60), 60\% EtOAc in $n$-hexane, $\mathrm{R}_{f}=0.39 ;{ }^{1} \mathrm{H} \mathrm{NMR}\left(300 \mathrm{MHz}, \mathrm{CDCl}_{3}\right) \delta 14.83(\mathrm{~s}, 0.49 \times 1 \mathrm{H}$, enol), 5.99-5.85 (m, 1H), $5.44(\mathrm{~s}, 0.49 \times 1 \mathrm{H}$, enol), 5.33-5.17 (m, 2H), 4.12-3.87 (m, 3H), $3.76(\mathrm{~d}, J=$ $16.1 \mathrm{~Hz}, 0.51 \times 1 \mathrm{H}$, keto), $3.63(\mathrm{~d}, J=16.1 \mathrm{~Hz}, 0.51 \times 1 \mathrm{H}$, keto), $3.01(\mathrm{~s}, 3 \mathrm{H}), 2.99(\mathrm{~s}$, $0.51 \times 3 \mathrm{H}$, keto), $2.98(\mathrm{~s}, 0.49 \times 3 \mathrm{H}$, enol), $1.40(\mathrm{~d}, J=6.6 \mathrm{~Hz}, 0.51 \times 3 \mathrm{H}$, keto $), 1.37$ $\left(\mathrm{d}, J=6.8 \mathrm{~Hz}, 0.49 \times 3 \mathrm{H}\right.$, enol); ${ }^{13} \mathrm{C} \mathrm{NMR}\left(75.5 \mathrm{MHz}, \mathrm{CDCl}_{3}\right) \delta 206.9,178.1,172.6$, $167.4,134.9,134.4,117.8,117.4,84.5,80.5,75.5,71.3,70.7,44.5,38.3,35.7,20.4$, 17.0; IR $\left(\mathrm{CH}_{2} \mathrm{Cl}_{2}\right)$ 3052, 2990, 2948, 1732, 1643, $1596 \mathrm{~cm}^{-1}$; LRMS (EI, $\left.20 \mathrm{eV}\right) \mathrm{m} / \mathrm{z}$ $199\left(\mathrm{M}^{+}, 3\right), 143$ (33), 127 (14), 114 (100); HRMS (EI) for $\mathrm{C}_{10} \mathrm{H}_{17} \mathrm{O}_{3} \mathrm{~N}\left(\mathrm{M}^{+}\right)$: calcd 199.1208, found 199.1211. 


\section{Preparation of compound 1i:}

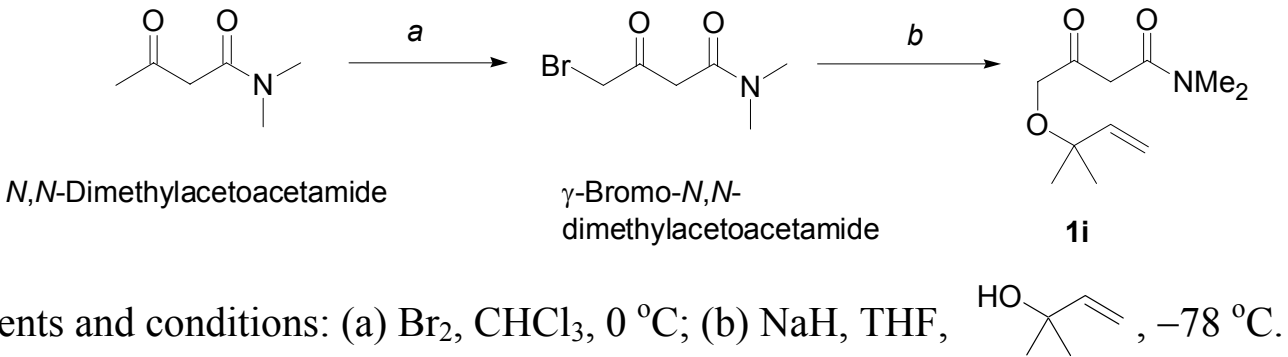

\section{Typical procedure for the preparation of 1i:}

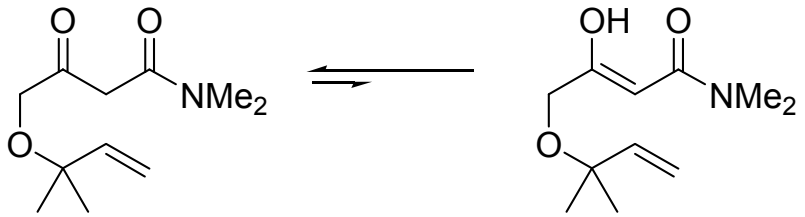

Keto $:$ Enol $=3.5: 1$

Step (a) $)^{7 \mathrm{a}}$ : To a solution of $N, N$-dimethylacetoacetamide $(20 \mathrm{~g}, 154.8 \mathrm{mmol})$ in chloroform $(300 \mathrm{~mL})$ over ice-water bath was added bromine $(9.1 \mathrm{~mL}, 177.6 \mathrm{mmol})$ dropwise with vigorous stirring. The mixture was stirred overnight at room temperature. The solvent was distilled off to give a crude product of $\gamma$-bromo- $N, N$ dimethylacetoacetamide.

Step $(b)^{7 b}$ : To a suspension of sodium hydride $(60 \%$ dispersion in oil, $724 \mathrm{mg}, 18.1$ mmol $)$ in THF $(60 \mathrm{~mL})$ was added the crude product of $\gamma$-bromo- $N, N$ dimethylacetoacetamide slowly at $-78{ }^{\circ} \mathrm{C}$. After $2 \mathrm{~h}$, a solution of alkoxide in THF [prepared from corresponding alcohol $(16.5 \mathrm{mmol})$ and sodium hydride $(18.1 \mathrm{mmol})$ in THF $(60 \mathrm{~mL})$ at $0{ }^{\circ} \mathrm{C}$ ] was cannulated to the reaction mixture at $-78{ }^{\circ} \mathrm{C}$. After $5 \mathrm{~h}$, the reaction was quenched by water $(10 \mathrm{~mL})$ and was extracted with ethyl acetate $(50$ $\mathrm{mL} \times 3)$. The combined organic layers were washed with brine $(50 \mathrm{~mL})$, dried over $\mathrm{Mg}_{2} \mathrm{SO}_{4}$ and concentrated in vacuo. The crude product was purified by flash column chromatography to give $\mathbf{1 i}(1.76 \mathrm{~g}, 8.3 \mathrm{mmol}, 50 \%)$ as a yellow oil; analytical TLC (silica gel 60), 50\% EtOAc in $n$-hexane, $\mathrm{R}_{f}=0.4 ;{ }^{1} \mathrm{H}$ NMR $\left(300 \mathrm{MHz}, \mathrm{CDCl}_{3}\right) \delta$ 14.83 (bs, $0.22 \times 1 \mathrm{H}$, enol), 5.84-5.75 (m, 1H), 5.48 (s, $0.22 \times 1 \mathrm{H}$, enol), 5.21-5.14 $(\mathrm{m}, 2 \mathrm{H}), 4.10(\mathrm{~s}, 0.78 \times 2 \mathrm{H}$, keto), $3.90(\mathrm{~s}, 0.22 \times 2 \mathrm{H}$, enol), $3.62(\mathrm{~s}, 0.78 \times 2 \mathrm{H}$, keto), $3.00(\mathrm{~s}, 4 \mathrm{H}$, keto + enol), $2.97(\mathrm{~s}, 2 \mathrm{H}$, keto + enol), $1.32(\mathrm{~s}, 0.22 \times 6 \mathrm{H}$, enol), $1.30(\mathrm{~s}$, 
$0.78 \times 6 \mathrm{H}$, keto) ${ }^{13} \mathrm{C} \mathrm{NMR}\left(75.5 \mathrm{MHz}, \mathrm{CDCl}_{3}\right) \delta 203.5,174.9,172.7,166.6,142.6$, $142.2,114.8,114.3,86.8,84.7,76.3,68.5,62.4,45.4,44.9,37.7,35.1,25.5,25.3$; IR $\left(\mathrm{CH}_{2} \mathrm{Cl}_{2}\right)$ 3051, 2990, 2929, 1738, 1645, $1514 \mathrm{~cm}^{-1}$; LRMS (EI, $\left.20 \mathrm{eV}\right) \mathrm{m} / \mathrm{z} 213\left(\mathrm{M}^{+}\right.$, 3), 154 (26), 144 (39), 114 (100); HRMS (EI) for $\mathrm{C}_{11} \mathrm{H}_{19} \mathrm{O}_{3} \mathrm{~N}\left(\mathrm{M}^{+}\right)$: calcd 213.1365, found 213.1365 .

\section{Ytterbium Triflate-Promoted Palladium(II)-Catalyzed Aerobic Oxidative} Cyclizations:

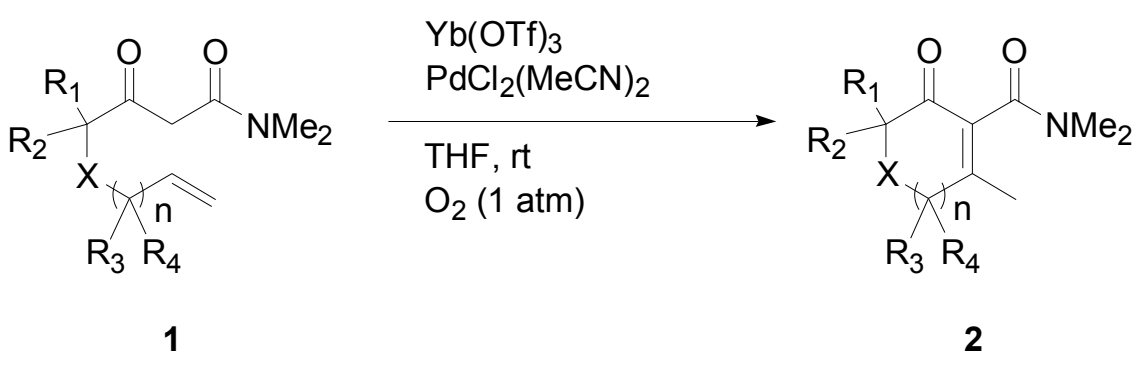

$$
\begin{aligned}
& \text { 1a,2a: } X=T s-N ; R_{1}=R_{2}=R_{3}=R_{4}=H ; n=1 \\
& \text { 1b,2b: } X=\text { Boc-N; } R_{1}=R_{2}=R_{3}=R_{4}=H ; n=1 \\
& \text { 1c,2c: } X=T s-N ; R_{1}=M e, R_{2}=H, R_{3}=R_{4}=H ; n=1 \\
& \text { 1d,2d: } X=T s-N ; R_{1}=R_{2}=R_{3}=R_{4}=H ; n=2 \\
& \text { 1e,2e: } X=T s-N ; R_{1}=R_{2}=R_{3}=R_{4}=H ; n=3 \\
& \text { 1f, 2f: } X=O ; R_{1}=R_{2}=R_{3}=R_{4}=H ; n=1 \\
& \text { 1g,2g: } X=O ; R_{1}=M e, R_{2}=H, R_{3}=R_{4}=H ; n=1 \\
& \text { 1h,2h: } X=O ; R_{1}=R_{2}=R_{3}=H, R_{4}=M e ; n=1 \\
& \text { 1i, 2i: } X=O ; R_{1}=R_{2}=H, R_{3}=R_{4}=M e ; n=1
\end{aligned}
$$

\section{Typical procedure for ytterbium triflate-promoted palladium(II)-catalyzed aerobic oxidative cyclization (Table 1):}

In a two-necked $25-\mathrm{mL}$ round-bottomed flask equipped with a magnetic stir bar, a mixture of $\mathrm{PdCl}_{2}\left(\mathrm{MeCN}_{2}(4 \mathrm{mg}, 0.015 \mathrm{mmol})\right.$ and anhydrous $\mathrm{Yb}(\mathrm{OTf})_{3}(93 \mathrm{mg}, 0.15$ mmol) in dry THF ( $5 \mathrm{~mL}$ ) solution was stirred for $5 \mathrm{~min}$ at room temperature. To this mixture was added a dry THF (5 mL) solution of 1 c $(53 \mathrm{mg}, 0.15 \mathrm{mmol})$ via syringe under oxygen atmosphere (1 atm). The addition rate $(5 \mathrm{~mL}$ over $3 \mathrm{hr}$ ) of 1c was controlled accurately by using a syringe pump. After the addition, the reaction progress was monitored by TLC analysis. The reaction was complete after $4 \mathrm{hr}$. The mixture was filtered through a short pad of silica gel (EtOAc as eluent) and 
concentrated in vacuo. The residue was purified by flash column chromatography (90-100\% EtOAc in $n$-hexane) to give $2 \mathrm{c}$ (47 $\mathrm{mg}, 90 \%$ yield).

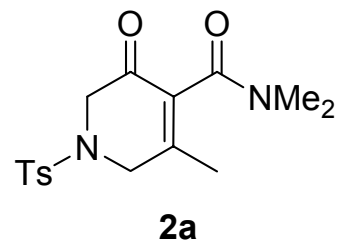

2a: Yield 98\%; an orange oil; analytical TLC (silica gel 60), 80\% EtOAc in $n$-hexane, $\mathrm{R}_{f}=0.15 ;{ }^{1} \mathrm{H} \mathrm{NMR}\left(300 \mathrm{MHz}, \mathrm{CDCl}_{3}\right) \delta 7.68(\mathrm{~d}, J=8.3 \mathrm{~Hz}, 2 \mathrm{H}), 7.37(\mathrm{~d}, J=8.1 \mathrm{~Hz}$, $2 \mathrm{H}), 4.21(\mathrm{~d}, J=18.2 \mathrm{~Hz}, 1 \mathrm{H}), 4.12(\mathrm{~d}, J=16.4 \mathrm{~Hz}, 1 \mathrm{H}), 3.63(\mathrm{~d}, J=18.2 \mathrm{~Hz}, 1 \mathrm{H})$, 3.44-3.38 (dd, $J=16.3,1.8 \mathrm{~Hz}, 1 \mathrm{H}), 3.02$ (s, 3H), 2.73 (s, 3H), 2.44 (s, 3H), 1.96 (s, $3 \mathrm{H}) ;{ }^{13} \mathrm{C} \mathrm{NMR}\left(75.5 \mathrm{MHz}, \mathrm{CDCl}_{3}\right) \delta 187.9,165.5,154.4,145.0,134.1,132.8,130.5$, 128.1, 52.2, 48.9, 37.9, 34.8, 21.9, 19.3; IR $\left(\mathrm{CH}_{2} \mathrm{Cl}_{2}\right)$ 3053, 3047, 2988, 2927, 1731, 1686, 1641, 1495, 1426, 1355, $1166 \mathrm{~cm}^{-1}$; LRMS (EI, $\left.20 \mathrm{eV}\right) \mathrm{m} / z 336\left(\mathrm{M}^{+}, 14\right), 181$ (72), 153 (36), 136 (100); HRMS (EI) for $\mathrm{C}_{16} \mathrm{H}_{20} \mathrm{O}_{4} \mathrm{~N}_{2} \mathrm{~S}\left(\mathrm{M}^{+}\right)$: calcd 336.1144, found 336.1139 .

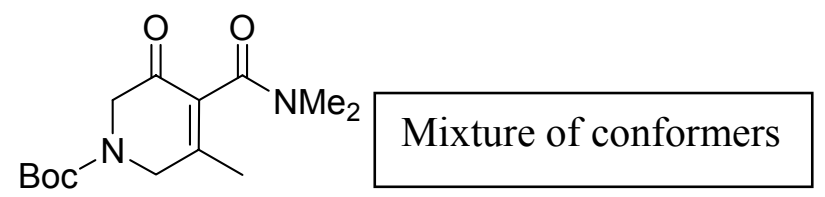

$2 b$

2b: Yield 68\%; a yellow oil; analytical TLC (silica gel 60), 80\% EtOAc in $n$-hexane, $\mathrm{R}_{f}=0.20 ;{ }^{1} \mathrm{H} \mathrm{NMR}\left(300 \mathrm{MHz}, \mathrm{CDCl}_{3}\right) \delta 4.53-3.85(\mathrm{~m}, 4 \mathrm{H}), 3.07(\mathrm{~s}, 1 \mathrm{H}), 2.98(\mathrm{~s}$, 2H), $2.87(\mathrm{~s}, 2 \mathrm{H}), 2.82(\mathrm{~s}, 1 \mathrm{H}), 2.08-1.79(\mathrm{~m}, 3 \mathrm{H}), 1.52-1.44(\mathrm{~m}, 9 \mathrm{H}),{ }^{13} \mathrm{C} \mathrm{NMR}(76$ $\left.\mathrm{MHz}, \mathrm{CDCl}_{3}\right) \delta 189.9,169.5,166.3,155.3,133.6,81.9,52.5,50.1,38.0,34.8,28.5$, 19.1; IR $\left(\mathrm{CH}_{2} \mathrm{Cl}_{2}\right)$ 3052, 2990, 2940, 1733, 1646, 1378, $1246 \mathrm{~cm}^{-1}$; LRMS (EI, $20 \mathrm{eV}$ ) $m / z 283\left(\mathrm{M}^{+}+\mathrm{H}, 18\right), 226$ (50), 183 (41), 180 (71), 136 (100); HRMS (EI) for $\mathrm{C}_{14} \mathrm{H}_{22} \mathrm{O}_{4} \mathrm{~N}_{2}\left(\mathrm{M}^{+}\right)$: calcd 282.1580, found 282.1580.

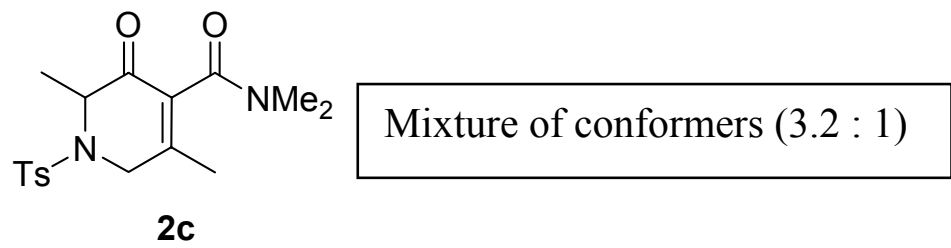


2c: Yield 90\%; a yellow oil; analytical TLC (silica gel 60), 80\% EtOAc in $n$-hexane, $\mathrm{R}_{f}=0.48 ;{ }^{1} \mathrm{H}$ NMR $\left(300 \mathrm{MHz}, \mathrm{CDCl}_{3}\right) \delta 7.69(\mathrm{~d}, J=8.3 \mathrm{~Hz}, 0.76 \times 2 \mathrm{H}$, major conformer), $7.64(\mathrm{~d}, J=8.3 \mathrm{~Hz}, 0.24 \times 2 \mathrm{H}$, minor conformer), 7.30 (d, $J=8.1 \mathrm{~Hz}$, 2H), 4.53-4.27 (m, 2H), 4.05-3.94 (m, 1H), 2.99 (s, 1H), $2.96(\mathrm{~s}, 2 \mathrm{H}), 2.73$ (s, $0.24 \times$ $3 \mathrm{H}$, minor conformer), $2.43(\mathrm{~s}, 1 \mathrm{H}), 2.40(\mathrm{~s}, 2 \mathrm{H}), 2.37(\mathrm{~s}, 0.76 \times 3 \mathrm{H}$, major conformer), $1.92(\mathrm{~s}, 0.76 \times 3 \mathrm{H}$, major conformer $), 1.87(\mathrm{~s}, 0.24 \times 3 \mathrm{H}$, minor conformer), $1.36(\mathrm{~d}, J=7.2 \mathrm{~Hz}, 0.24 \times 3 \mathrm{H}$, minor conformer), 1.29 (d, $J=7.2 \mathrm{~Hz}$, $0.76 \times 3 \mathrm{H}$, major conformer); ${ }^{13} \mathrm{C} \mathrm{NMR}\left(75.5 \mathrm{MHz}, \mathrm{CDCl}_{3}\right) \delta 191.5,165.6,153.2$, 144.4, 137.0, 132.3, 130.5, 127.4, 56.4, 44.7, 37.4, 34.7, 21.8, 18.8, 15.3; IR $\left(\mathrm{CH}_{2} \mathrm{Cl}_{2}\right)$ 3051, 2988, 2923, 1738, 1683, 1643, 1495, 1436, 1403, 1246, $1163 \mathrm{~cm}^{-1}$; LRMS (EI, $20 \mathrm{eV}) \mathrm{m} / z 350\left(\mathrm{M}^{+}, 12\right), 209$ (7), 195 (38), 167 (23), 150 (100); HRMS (EI) for $\mathrm{C}_{17} \mathrm{H}_{22} \mathrm{O}_{4} \mathrm{~N}_{2} \mathrm{~S}\left(\mathrm{M}^{+}\right)$: calcd 350.1300, found 350.1296.

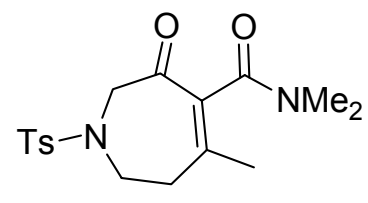

2d

2d: Yield 91\%; a yellow oil; analytical TLC (silica gel 60), 80\% EtOAc in $n$-hexane, $\mathrm{R}_{f}=0.13 ;{ }^{1} \mathrm{H} \mathrm{NMR}\left(500 \mathrm{MHz}\right.$ at $\left.-30{ }^{\circ} \mathrm{C}, \mathrm{CDCl}_{3}\right) \delta 7.70(\mathrm{~d}, J=8.2 \mathrm{~Hz}, 2 \mathrm{H}), 7.39(\mathrm{~d}, J$ $=8.2 \mathrm{~Hz}, 2 \mathrm{H}), 4.42(\mathrm{~d}, J=18.1 \mathrm{~Hz}, 1 \mathrm{H}), 3.90-3.85(\mathrm{~m}, 1 \mathrm{H}), 3.56(\mathrm{~d}, J=18.2 \mathrm{~Hz}$, $1 \mathrm{H}), 3.10(\mathrm{~s}, 3 \mathrm{H}), 3.08-3.03(\mathrm{~m}, 1 \mathrm{H}), 3.02(\mathrm{~s}, 3 \mathrm{H}), 2.76-2.63(\mathrm{~m}, 2 \mathrm{H}), 2.48(\mathrm{~s}, 3 \mathrm{H})$, $2.09(\mathrm{~s}, 3 \mathrm{H}) ;{ }^{13} \mathrm{C} \mathrm{NMR}\left(126 \mathrm{MHz}\right.$ at $\left.-30{ }^{\circ} \mathrm{C}, \mathrm{CDCl}_{3}\right) \delta 197.2,167.3,151.9,144.4$, 136.0, 133.4, 130.0, 126.9, 55.9, 44.3, 38.0, 34.7, 34.4, 24.2, 21.7; IR $\left(\mathrm{CH}_{2} \mathrm{Cl}_{2}\right)$ 3051, 2990, 2948, 1733, 1677, 1637, 1420, $1164 \mathrm{~cm}^{-1}$; LRMS (EI, $\left.20 \mathrm{eV}\right) \mathrm{m} / \mathrm{z} 350\left(\mathrm{M}^{+}, 21\right)$, 237 (69), 195 (100), 150 (67); HRMS (EI) for $\mathrm{C}_{17} \mathrm{H}_{22} \mathrm{O}_{4} \mathrm{~N}_{2} \mathrm{~S}\left(\mathrm{M}^{+}\right)$: calcd 350.1300, found 350.1297 .

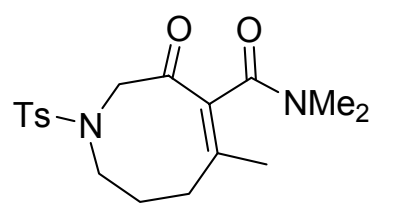

Mixture of conformers

$2 e$

2e: Yield 62\%; a brown oil; analytical TLC (silica gel 60), 80\% EtOAc in $n$-hexane, $\mathrm{R}_{f}=0.15 ;{ }^{1} \mathrm{H}$ NMR $\left(300 \mathrm{MHz}, \mathrm{CD}_{2} \mathrm{Cl}_{2}\right) \delta$ 7.66-7.58 (m, 2H), 7.29-7.22 (m, 2H), 
$3.82(\mathrm{~s}, 1 \mathrm{H}), 3.57(\mathrm{~m}, 1 \mathrm{H}), 3.43-3.38(\mathrm{~m}, 1 \mathrm{H}), 3.13(\mathrm{t}, J=5.8 \mathrm{~Hz}, 1 \mathrm{H}), 2.98-2.79(\mathrm{~m}$, $6 \mathrm{H}), 2.35$ (s, 3H), 2.33-2.27 (m, 2H), $1.70(\mathrm{~s}, 3 \mathrm{H}), 1.34-1.13(\mathrm{~m}, 2 \mathrm{H}) ;{ }^{13} \mathrm{C}$ NMR $(76$ $\left.\mathrm{MHz}, \mathrm{CD}_{2} \mathrm{Cl}_{2}\right) \delta 197.5,167.2,155.2,144.1,136.3,135.2,130.0,127.4,55.7,44.9$, 38.0, 35.4, 31.7, 30.8, 21.5, 11.8; IR $\left(\mathrm{CH}_{2} \mathrm{Cl}_{2}\right)$ 3050, 2985, 2940, 1744, 1688, 1637 , 1495, 1420, $1164 \mathrm{~cm}^{-1}$; LRMS (EI, $\left.20 \mathrm{eV}\right) \mathrm{m} / z 364$ (M+ 23), 251 (88), 209 (100), 164 (99), 155 (33); HRMS (EI) for $\mathrm{C}_{18} \mathrm{H}_{24} \mathrm{O}_{4} \mathrm{~N}_{2} \mathrm{~S}\left(\mathrm{M}^{+}\right)$: calcd 364.1464, found 364.1447.<smiles>CC1=C(C(=O)N(C)C)C(=O)COC1</smiles>

$2 f$

2f: Yield 97\%; a brown oil; analytical TLC (silica gel 60), 80\% EtOAc in $n$-hexane, $\mathrm{R}_{f}=0.12 ;{ }^{1} \mathrm{H}$ NMR $\left(400 \mathrm{MHz}, \mathrm{CDCl}_{3}\right) \delta 4.37(\mathrm{~d}, J=18.0 \mathrm{~Hz}, 1 \mathrm{H}), 4.32(\mathrm{~d}, J=17.5$ $\mathrm{Hz}, 1 \mathrm{H}), 4.21(\mathrm{~d}, J=16.1 \mathrm{~Hz}, 1 \mathrm{H}), 4.13$ (d, $J=16.1 \mathrm{~Hz}, 1 \mathrm{H}), 3.08$ (s, 3H), 2.91 (s, $3 \mathrm{H}), 1.95(\mathrm{~s}, 3 \mathrm{H}) ;{ }^{13} \mathrm{C} \mathrm{NMR}\left(75.5 \mathrm{MHz}, \mathrm{CDCl}_{3}\right) \delta 190.6,165.6,157.4,132.8,71.5$, 68.9, 38.1, 34.9, 17.4; IR $\left(\mathrm{CH}_{2} \mathrm{Cl}_{2}\right)$ 3051, 2985, 2940, 1733, 1682, $1639 \mathrm{~cm}^{-1}$; LRMS (EI, $20 \mathrm{eV}) \mathrm{m} / z 183\left(\mathrm{M}^{+}, 31\right), 153$ (100), 139 (20); HRMS (EI) for $\mathrm{C}_{9} \mathrm{H}_{13} \mathrm{O}_{3} \mathrm{~N}\left(\mathrm{M}^{+}\right)$: calcd 183.0895, found 183.0889.

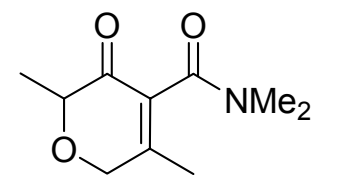

Mixture of conformer $(1.9: 1)$

$2 \mathrm{~g}$

2g: Yield 96\%; a brown oil; analytical TLC (silica gel 60), 80\% EtOAc in $n$-hexane, $\mathrm{R}_{f}=0.21 ;{ }^{1} \mathrm{H}$ NMR $\left(300 \mathrm{MHz}, \mathrm{CDCl}_{3}\right) \delta 4.45-4.29(\mathrm{~m}, 2 \mathrm{H}), 4.16-4.06(\mathrm{~m}, 1 \mathrm{H}), 3.08$ (s, 3H), $2.91(\mathrm{~s}, 2 \mathrm{H}), 2.87(\mathrm{~s}, 1 \mathrm{H}), 1.93(\mathrm{~s}, 2 \mathrm{H}), 1.92(\mathrm{~s}, 1 \mathrm{H}), 1.42(\mathrm{~d}, J=6.6 \mathrm{~Hz}, 3 \mathrm{H})$;

${ }^{13} \mathrm{C}$ NMR $\left(100 \mathrm{MHz}, \mathrm{CDCl}_{3}\right) \delta 192.6,166.1,156.7,156.3,132.3,76.5,76.0,68.7$, 67.0, 38.2, 38.0, 34.8, 17.3, 17.2, 15.7, 15.5; IR $\left(\mathrm{CH}_{2} \mathrm{Cl}_{2}\right)$ 3051, 2996, 2940, 1788, 
1732, 1688, $1639 \mathrm{~cm}^{-1}$; LRMS (EI, $\left.20 \mathrm{eV}\right) \mathrm{m} / z 197\left(\mathrm{M}^{+}, 26\right), 167$ (100), 153 (22), 136 (21); HRMS (EI) for $\mathrm{C}_{10} \mathrm{H}_{15} \mathrm{O}_{3} \mathrm{~N}\left(\mathrm{M}^{+}\right)$: calcd 197.1052, found 197.1035.<smiles>CC(=O)C1=C(C)C(C)OCC1=O</smiles>

$2 \mathrm{~h}$

2h: Yield 98\%; a brown oil; analytical TLC (silica gel 60), 80\% EtOAc in $n$-hexane, $\mathrm{R}_{f}=0.18 ;{ }^{1} \mathrm{H}$ NMR $\left(500 \mathrm{MHz}\right.$ at $\left.-30{ }^{\circ} \mathrm{C}, \mathrm{CDCl}_{3}\right) \delta 4.52-4.46(\mathrm{~m}, 1 \mathrm{H}), 4.39-4.32(\mathrm{~m}$, 1H), 4.20-4.17 (m, 1H), 3.12 (s, 3H), 2.98 and 2.94 (s, 3H), 1.98 (s, 3H), 1.53-1.50 $(\mathrm{m}, 3 \mathrm{H}) ;{ }^{13} \mathrm{C} \mathrm{NMR}\left(126 \mathrm{MHz}\right.$ at $\left.-30{ }^{\circ} \mathrm{C}, \mathrm{CDCl}_{3}\right) \delta 191.2,191.1,165.7,165.5,131.9$, 131.3, 125.9, 73.0, 72.5, 70.5, 67.4, 38.0, 34.5, 18.6, 18.1, 17.5, 16.6; IR $\left(\mathrm{CH}_{2} \mathrm{Cl}_{2}\right)$ 3051, 2989, 2948, 1734, 1682, $1639 \mathrm{~cm}^{-1}$; LRMS (ESI) $m / z 198\left(\mathrm{M}^{+}+\mathrm{H}, 90\right)$; HRMS (EI) for $\mathrm{C}_{10} \mathrm{H}_{15} \mathrm{O}_{3} \mathrm{~N}\left(\mathrm{M}^{+}\right)$: calcd 197.1052, found 197.1054.<smiles>CCC1=C(C(=O)N(C)C)C(=O)COC1(C)C</smiles>

$2 i$

2i: Yield 92\%; a brown oil; analytical TLC (silica gel 60), 80\% EtOAc in $n$-hexane, $\mathrm{R}_{f}=0.24 ;{ }^{1} \mathrm{H}$ NMR $\left(400 \mathrm{MHz}, \mathrm{CDCl}_{3}\right) \delta 4.30(\mathrm{~d}, J=17.1 \mathrm{~Hz}, 1 \mathrm{H}), 4.21(\mathrm{~d}, J=17.1$ $\mathrm{Hz}, 1 \mathrm{H}), 3.07$ (s, 3H), 2.88 (s, 3H), 1.92 (s, 3H), 1.48 (s, 3H), 1.47 (s, 3H); ${ }^{13} \mathrm{C} \mathrm{NMR}$ $\left(100 \mathrm{MHz} \mathrm{CDCl}_{3}\right) \delta 191.2,166.3,162.5,131.9,75.5,66.8,37.9,34.8,26.1,23.6$, 17.6; IR $\left(\mathrm{CH}_{2} \mathrm{Cl}_{2}\right)$ 3051, 2988, 2929, 1732, 1684, $1639 \mathrm{~cm}^{-1}$; LRMS (EI, $\left.20 \mathrm{eV}\right) \mathrm{m} / \mathrm{z}$ $211\left(\mathrm{M}^{+}, 63\right), 167$ (52), 153 (100), 110 (50); HRMS (EI) for $\mathrm{C}_{11} \mathrm{H}_{17} \mathrm{O}_{3} \mathrm{~N}\left(\mathrm{M}^{+}\right)$: calcd 211.1208 , found 211.1201 .

Preparation of compounds 1fa:

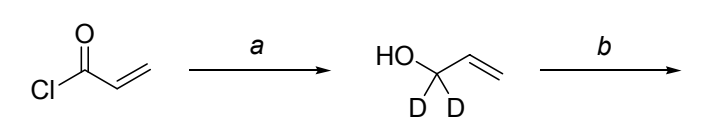

acryloyl chloride

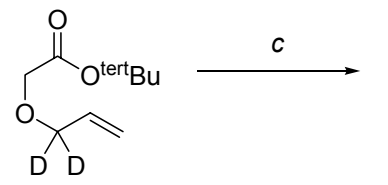

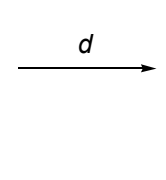

$8 \mathrm{fa}$<smiles>[2H]C([2H])(O)CC(=O)CC(C)=O</smiles>

$1 \mathrm{fa}$ 
Reagents and conditions: (a) $\mathrm{LiAlD}_{4}, \mathrm{Et}_{2} \mathrm{O}, 0{ }^{\circ} \mathrm{C}$ to $\mathrm{rt}$ then quench by $\mathrm{H}_{2} \mathrm{O}$; (b) tertButyl bromoacetate, $50 \%$ aqueous $\mathrm{NaOH},(n-\mathrm{Bu})_{4} \mathrm{NHSO}_{4}$, benzene, $0{ }^{\circ} \mathrm{C}$; (c) $\mathrm{SOCl}_{2}$, $\mathrm{MeOH}, 0{ }^{\circ} \mathrm{C}$; (d) $N, N$-dimethylacetamide, LDA, THF, $-78{ }^{\circ} \mathrm{C}$.

\section{Typical procedure for the preparation of 8fa:}

To a solution of $\mathrm{LiAlD}_{4}(2.83 \mathrm{~g}, 67.3 \mathrm{mmol}$; Aldrich, 98\% deuterium) in dry diethyl ether $(140 \mathrm{~mL})$ was stirred at $0{ }^{\circ} \mathrm{C}$. The mixture was slowly added acryloyl chloride $(8.2 \mathrm{~mL}, 100.9 \mathrm{mmol})$ as an ethereal solution $(40 \mathrm{~mL})$ through addition funnel over 30 min. The resulting solution was warmed to room temperature and stirred for $14 \mathrm{~h}$. The mixture was cooled to $0{ }^{\circ} \mathrm{C}$ and $\mathrm{H}_{2} \mathrm{O}(2.8 \mathrm{~mL})$ was slowly added over a 10 -min period. After stirring for another $15 \mathrm{~min}, 15 \%$ aqueous $\mathrm{NaOH}$ solution $(2.8 \mathrm{~mL})$ and then $\mathrm{H}_{2} \mathrm{O}(2.8 \mathrm{~mL})$ were added. The grey slurry was filtered via a short pad of celite and was washed by $\mathrm{Et}_{2} \mathrm{O}(150 \mathrm{~mL})$. The filtrate was concentrated in vacuo to give allyl-1$d_{2}$ alcohol as a yellow oil. ${ }^{8}$

The crude product (allyl-1- $d_{2}$ alcohol) was converted to 8 fa in 22\% yield (over 3 steps from acryloyl chloride) employing the procedure for the preparation of $\mathbf{8}$.

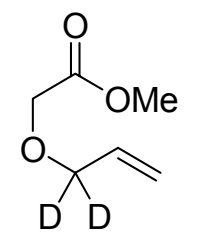

$8 f a$

$>95 \%$ deuterium content by ${ }^{1} \mathrm{H}$ NMR

8fa: a yellow oil; ${ }^{1} \mathrm{H} \mathrm{NMR}\left(300 \mathrm{MHz}, \mathrm{CDCl}_{3}\right) \delta 5.92(\mathrm{dd}, J=17.2,10.4 \mathrm{~Hz}, 1 \mathrm{H})$, 5.35-5.23 (m, 2H), $4.10(\mathrm{~s}, 2 \mathrm{H}), 3.77(\mathrm{~s}, 3 \mathrm{H}) ;{ }^{13} \mathrm{C} \mathrm{NMR}\left(75 \mathrm{MHz}, \mathrm{CDCl}_{3}\right) \delta 171.1$, 133.9, 118.8, $73.4\left(\mathrm{~m},-\mathrm{CD}_{2}-\right), 67.3,52.1$.

1fa was prepared in $76 \%$ yield from $\mathbf{8 f a}$ employing the procedure for the preparation of 1 .

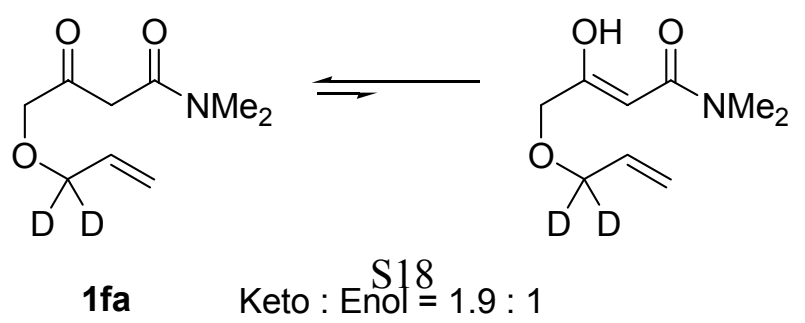


$>95 \%$ deuterium content by ${ }^{1} \mathrm{H}$ NMR

1fa: a yellow oil; ${ }^{1} \mathrm{H}$ NMR (400 MHz, $\left.\mathrm{CDCl}_{3}\right) \delta 14.88(\mathrm{~s}, 0.35 \times 1 \mathrm{H}$, enol), 5.93-5.86 (m, 1H), $5.46(\mathrm{~s}, 0.35 \times 1 \mathrm{H}$, enol), 5.34-5.22 (m, 2H), $4.18(\mathrm{~s}, 0.65 \times 2 \mathrm{H}$, keto), 4.03 $(\mathrm{s}, 0.35 \times 2 \mathrm{H}$, enol), $3.60(\mathrm{~s}, 0.65 \times 2 \mathrm{H}$, keto $), 3.01(\mathrm{~s}, 4 \mathrm{H}$, keto + enol), $2.97(\mathrm{~s}, 2 \mathrm{H}$, keto + enol); ${ }^{13} \mathrm{C} \mathrm{NMR}\left(100 \mathrm{MHz}, \mathrm{CDCl}_{3}\right) \delta 203.2,173.9,172.3,166.9,134.3,133.9$, 118.5, 118.1, 85.6, 75.1, 71.7 (m, - $\left.\mathrm{CD}_{2}-\right)$, 69.4, 45.7, 38.2, 35.7; LRMS (EI, $20 \mathrm{eV}$ ) $m / z 187\left(\mathrm{M}^{+}, 6\right), 144(64)$; HRMS (EI) for $\mathrm{C}_{9} \mathrm{H}_{13} \mathrm{D}_{2} \mathrm{O}_{3} \mathrm{~N}\left(\mathrm{M}^{+}\right)$: calcd 187.1178, found 187.1180.

\section{Preparation of compound 1fb:}

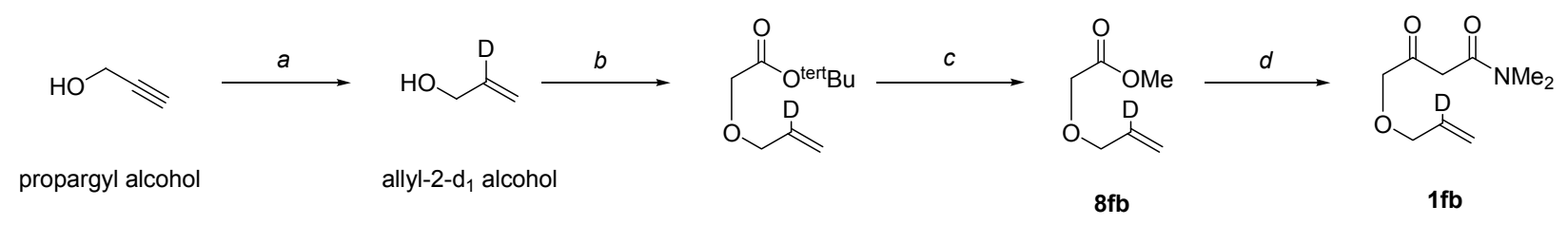

Reagents and conditions: (a) $\mathrm{LiAlD}_{4}, \mathrm{Et}_{2} \mathrm{O}, 0{ }^{\circ} \mathrm{C}$ to $\mathrm{rt}$ then quench by $\mathrm{H}_{2} \mathrm{O}$; (b) tertButyl bromoacetate, $50 \%$ aqueous $\mathrm{NaOH},(n-\mathrm{Bu})_{4} \mathrm{NHSO}_{4}$, benzene, $0{ }^{\circ} \mathrm{C}$; (c) $\mathrm{SOCl}_{2}$, $\mathrm{MeOH}, 0{ }^{\circ} \mathrm{C}$; (d) $N, N$-dimethylacetamide, LDA, THF, $-78^{\circ} \mathrm{C}$.

\section{Typical procedure for the preparation of $8 \mathrm{fb}$ :}

To a solution of $\mathrm{LiAlD}_{4}(2.00 \mathrm{~g}, 47.6 \mathrm{mmol}$; Aldrich, 98\% deuterium) in dry diethyl ether $(140 \mathrm{~mL})$ was stirred at $-10{ }^{\circ} \mathrm{C}$. The mixture was slowly added propargyl alcohol (2.67 g, $47.6 \mathrm{mmol})$ as an ethereal solution $(30 \mathrm{~mL})$ through addition funnel over $30 \mathrm{~min}$. The resulting solution was warmed to room temperature and stirred for $14 \mathrm{~h}$. The mixture was cooled to $0{ }^{\circ} \mathrm{C}$ and $\mathrm{H}_{2} \mathrm{O}(2.0 \mathrm{~mL})$ was slowly added over a 10 min period. After stirring for another $15 \mathrm{~min}, 15 \%$ aqueous $\mathrm{NaOH}$ solution $(2.0 \mathrm{~mL})$ 
and then $\mathrm{H}_{2} \mathrm{O}(2.0 \mathrm{~mL})$ were added. The grey slurry was filtered via a short pad of celite and was washed by $\mathrm{Et}_{2} \mathrm{O}(100 \mathrm{~mL})$. The filtrate was concentrated in vacuo to give allyl-2- $d_{1}$ alcohol as a yellow oil. ${ }^{9}$

The crude product (allyl-2- $d_{1}$ alcohol) was converted to $\mathbf{8 f b}$ in $49 \%$ yield (over 3 steps from propargyl alcohol) employing the procedure for the preparation of $\mathbf{8}$.<smiles>C=C(COCC(=O)OC)OC</smiles>

$8 \mathrm{fb}$

95\% deuterium content by ${ }^{1} \mathrm{H}$ NMR

8fb: a yellow oil; ${ }^{1} \mathrm{H}$ NMR $\left(300 \mathrm{MHz}, \mathrm{CDCl}_{3}\right) \delta 5.18(\mathrm{~s}, 1 \mathrm{H}), 5.11(\mathrm{~s}, 1 \mathrm{H}), 3.98(\mathrm{~s}$, $4 \mathrm{H}), 3.64(\mathrm{~s}, 3 \mathrm{H}) ;{ }^{13} \mathrm{C} \mathrm{NMR}\left(75 \mathrm{MHz}, \mathrm{CDCl}_{3}\right) \delta 170.8,133.5\left(\mathrm{t}, J_{\mathrm{CD}}=23.9 \mathrm{~Hz}\right)$, 118.0, 72.3, 67.1, 51.7 .

$\mathbf{1 f b}$ was isolated in $91 \%$ yield from $\mathbf{8 f b}$ employing the procedure for the preparation of 1 .

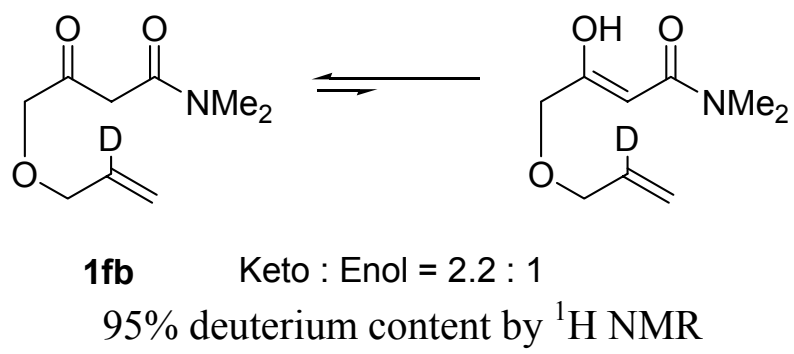

1fb: a yellow oil; ${ }^{1} \mathrm{H}$ NMR $\left(300 \mathrm{MHz}, \mathrm{CDCl}_{3}\right) \delta 14.88(\mathrm{~s}, 0.31 \times 1 \mathrm{H}$, enol), $5.46(\mathrm{~s}$, $0.31 \times 1 \mathrm{H}$, enol), $5.30(\mathrm{~s}, 1 \mathrm{H}), 5.23(\mathrm{~s}, 1 \mathrm{H}), 4.29-4.24(\mathrm{~m}, 1 \mathrm{H}), 4.18(\mathrm{~s}, 0.69 \times 1 \mathrm{H}$, keto), $4.13(\mathrm{~s}, 0.31 \times 1 \mathrm{H}$, enol), $4.07(\mathrm{~s}, 0.69 \times 2 \mathrm{H}$, keto $), 4.03(\mathrm{~s}, 0.31 \times 2 \mathrm{H}$, enol), $3.60\left(\mathrm{~s}, 0.69 \times 2 \mathrm{H}\right.$, keto), $3.01(\mathrm{~s}, 4 \mathrm{H}$, keto $+\mathrm{enol}), 2.97\left(\mathrm{~s}, 2 \mathrm{H}\right.$, keto + enol); ${ }^{13} \mathrm{C}$ $\operatorname{NMR}\left(75.5 \mathrm{MHz}, \mathrm{CDCl}_{3}\right) \delta 203.2,173.9,172.3,166.9,133.7\left(\mathrm{t}, J_{\mathrm{CD}}=23.9 \mathrm{~Hz}\right.$ ), 118.2, 117.8, 85.6, 75.2, 72.6, 72.3, 69.5, 58.9, 45.7, 38.2, 35.7; LRMS (EI, $20 \mathrm{eV}$ ) $m / z 186\left(\mathrm{M}^{+}, 1\right), 129$ (78), 114 (100); HRMS (EI) for $\mathrm{C}_{9} \mathrm{H}_{14} \mathrm{DO}_{3} \mathrm{~N}\left(\mathrm{M}^{+}\right)$: calcd 186.1115, found 186.1099 . 
Typical procedure for the aerobic oxidative cyclization of 1 fa and $1 \mathrm{fb}$ (eq 2 and 3):
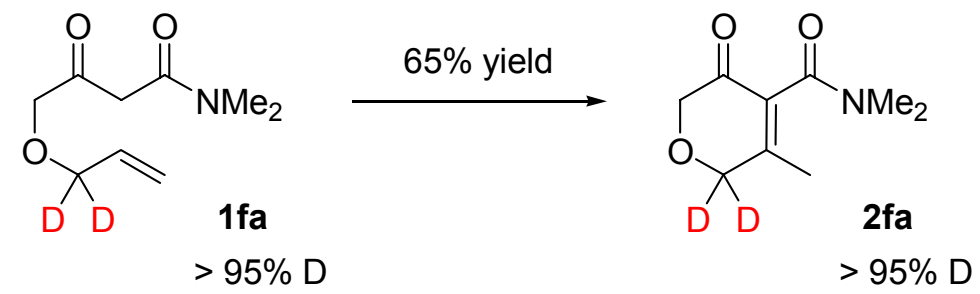

$$
\text { Conditions: } \begin{aligned}
& \mathrm{Yb}(\mathrm{OTf})_{3}(30 \mathrm{~mol} \%) \\
& \mathrm{PdCl}_{2}(\mathrm{MeCN})_{2}(10 \mathrm{~mol} \%) \\
& \mathrm{THF}, \mathrm{O}_{2}(1 \mathrm{~atm}), \mathrm{rt}
\end{aligned}
$$

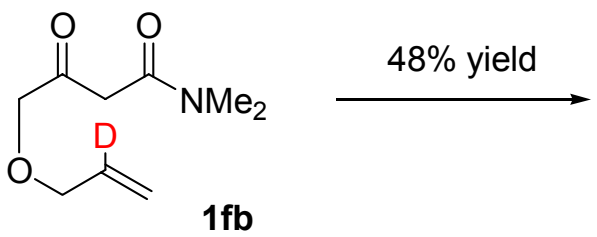

$95 \%$ D<smiles>[2H]CC1=C(C(=O)NC)C(=O)COC1</smiles>

$90 \%$ D

$\mathbf{2 f a}$ and $\mathbf{2} \mathbf{f b}$ were obtained employing the procedure for the preparation of $\mathbf{2} \mathbf{a}-\mathbf{i}$ except that the loading of $\mathrm{Yb}(\mathrm{OTf})_{3}$ was $30 \mathrm{~mol} \%$. 
Figure S1. ${ }^{1} \mathrm{H}$ NMR Spectra of $\mathbf{2 f}, \mathbf{2} \mathbf{f a}$ and $\mathbf{2 f b}$
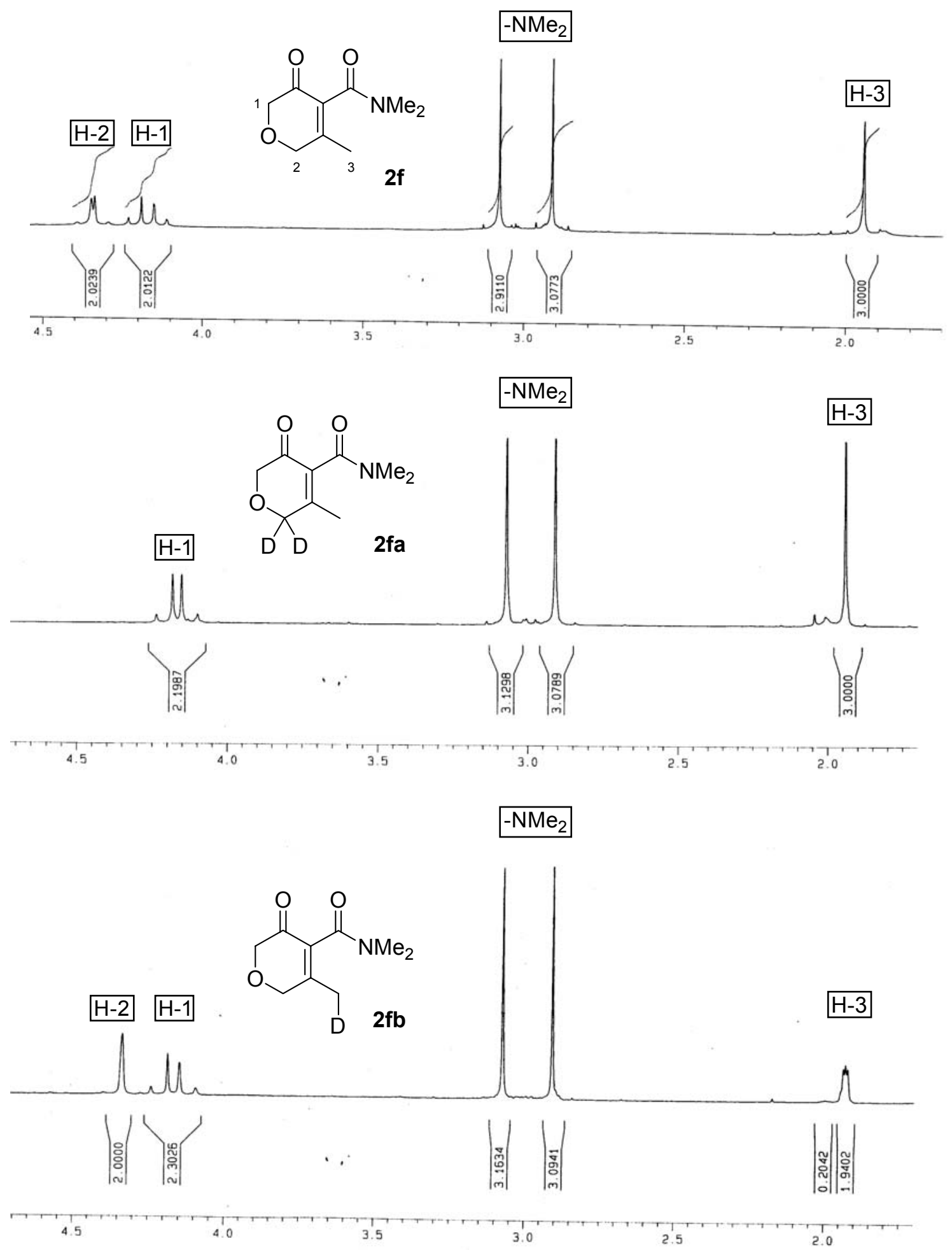
Figure S2. ${ }^{13} \mathrm{C}$ NMR Spectra of $\mathbf{2 f}, \mathbf{2 f a}$ and $\mathbf{2 f b}$
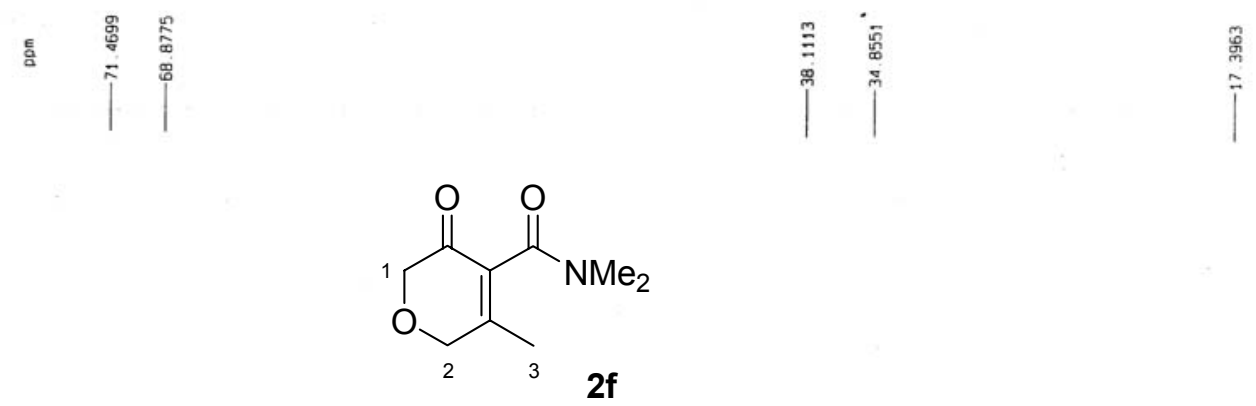

C-1 C-2

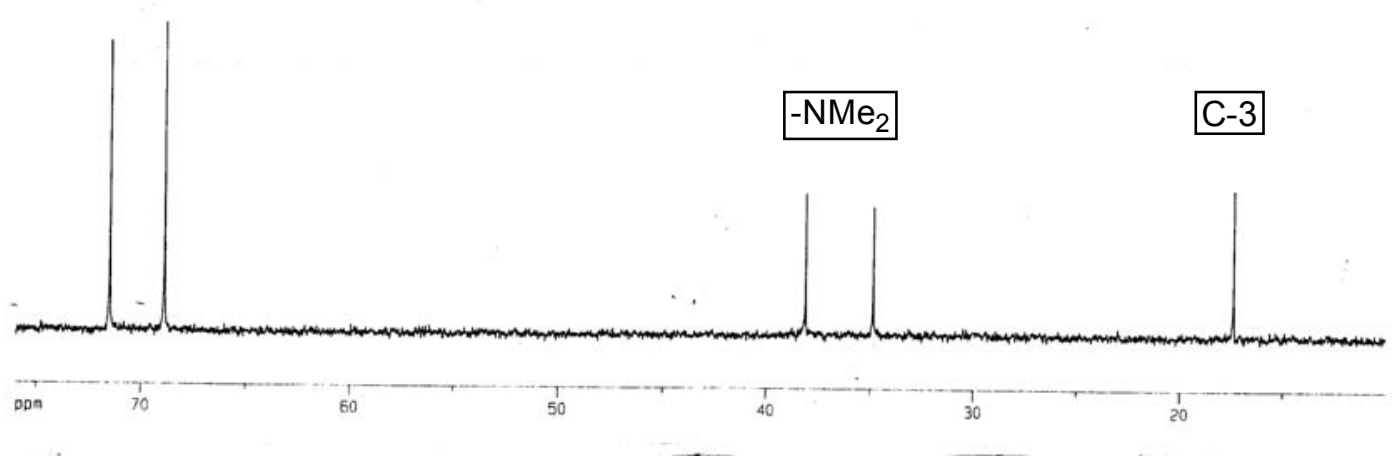

⿷匚⿱艹⿵\zh22⿷匚⿳丨コ丨
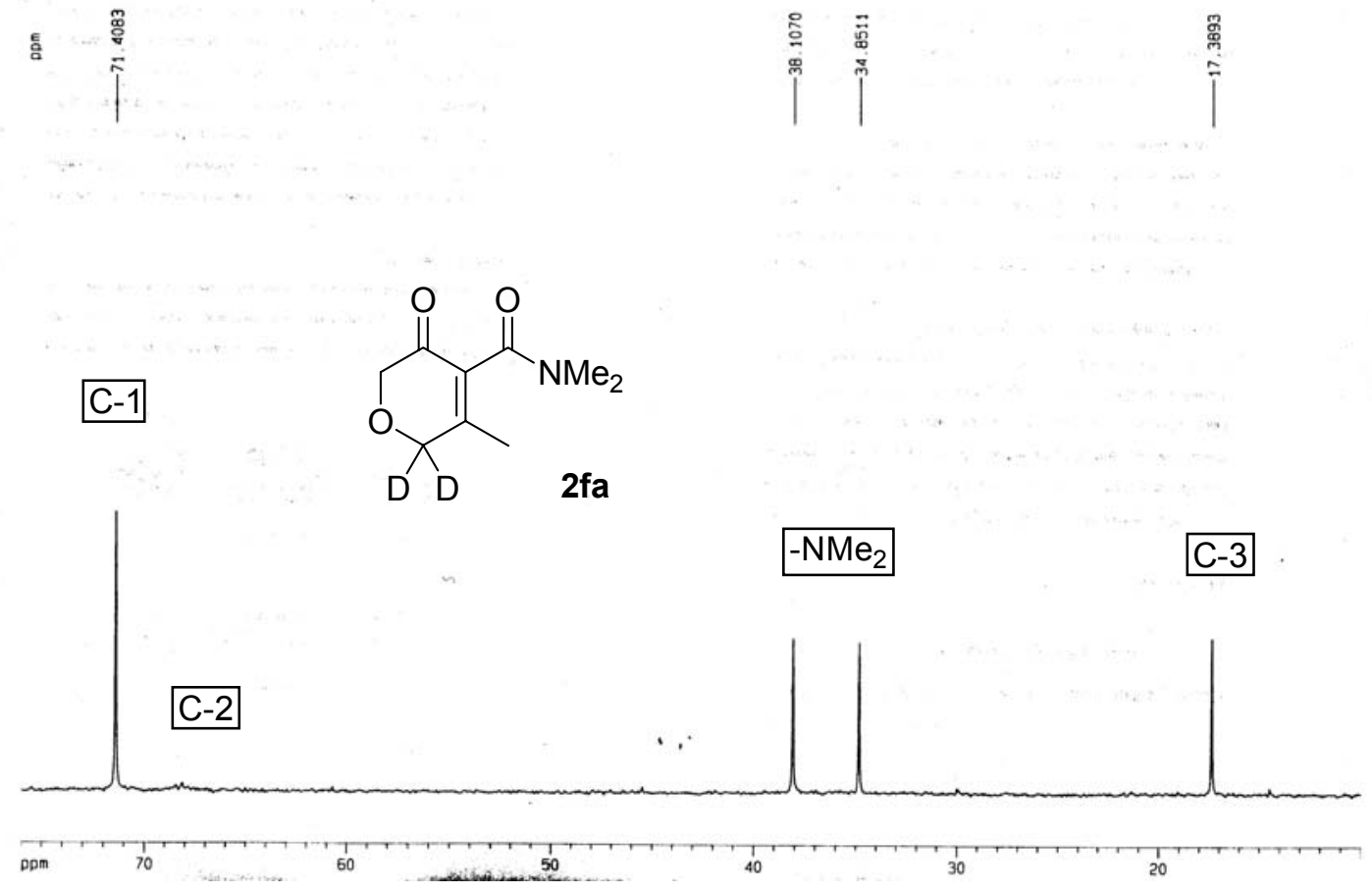


\section{Expanded Region of ${ }^{13} \mathrm{C}$ NMR Spectrum of $2 \mathrm{fa}$}

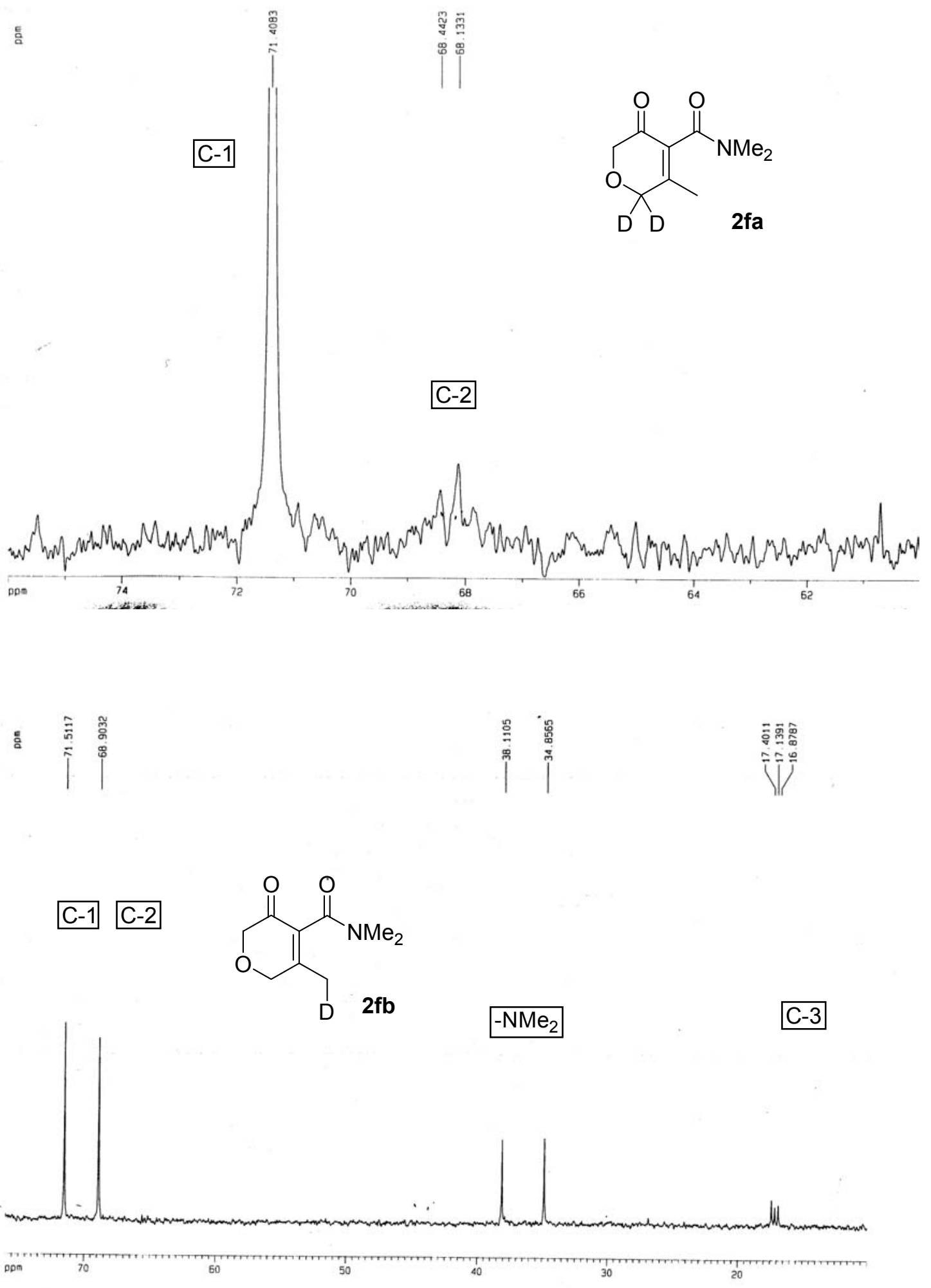


2fa: Yield 65\%; >95\% deuterium content; a brown oil; ${ }^{1} \mathrm{H}$ NMR $\left(300 \mathrm{MHz}, \mathrm{CDCl}_{3}\right) \delta$ $4.21(\mathrm{~d}, J=16.1 \mathrm{~Hz}, 1 \mathrm{H}), 4.13$ (d, $J=16.1 \mathrm{~Hz}, 1 \mathrm{H}), 3.07$ (s, 3H), 2.91 (s, 3H), 1.95 (s, $3 \mathrm{H}) ;{ }^{13} \mathrm{C}$ NMR $\left(75.5 \mathrm{MHz}, \mathrm{CDCl}_{3}\right) \delta 190.7,165.6,157.4,132.8,71.4,68.1(\mathrm{~m}$, $-\mathrm{CD}_{2}-$ ), 38.1, 34.9, 17.4; LRMS (EI, $\left.20 \mathrm{eV}\right) \mathrm{m} / z 185\left(\mathrm{M}^{+}, 7\right), 153$ (100); HRMS (EI) for $\mathrm{C}_{9} \mathrm{H}_{11} \mathrm{D}_{2} \mathrm{O}_{3} \mathrm{~N}\left(\mathrm{M}^{+}\right)$: calcd 185.1021, found 183.1029. See Figure S1 and S2.

2fb: Yield 48\%; 90\% deuterium content; a brown oil; ${ }^{1} \mathrm{H}$ NMR $\left(300 \mathrm{MHz}, \mathrm{CDCl}_{3}\right) \delta$ 4.34 (s, 2H), 4.22 (d, $J=16.1 \mathrm{~Hz}, 1 \mathrm{H}), 4.13$ (d, $J=16.1 \mathrm{~Hz}, 1 \mathrm{H}), 3.08$ (s, 3H), 2.91 (s, $3 \mathrm{H}), 1.93\left(\mathrm{t}, J_{\mathrm{HD}}=2.3 \mathrm{~Hz}, 2 \mathrm{H}\right) ;{ }^{13} \mathrm{C} \mathrm{NMR}\left(75.5 \mathrm{MHz}, \mathrm{CDCl}_{3}\right) \delta 190.6,165.6,157.2$, 133.0, 71.5, 68.9, 38.1, 34.9, 17.1 (t, $\left.J_{\mathrm{CD}}=19.8 \mathrm{~Hz},-\mathrm{CH}_{2} \mathrm{D}\right)$; LRMS (EI, $\left.20 \mathrm{eV}\right) \mathrm{m} / \mathrm{z}$ $184\left(\mathrm{M}^{+}, 14\right), 154$ (100); HRMS (EI) for $\mathrm{C}_{9} \mathrm{H}_{12} \mathrm{DO}_{3} \mathrm{~N}\left(\mathrm{M}^{+}\right)$: calcd 184.0958, found 184.0962. See Figure S1 and S2.

\section{References:}

1) Zhu, S.; Xu, Y. Tetrahedron 2001, 57, 3909.

2) Roos, E. C.; Bernabe, P.; Hiemstra, H.; Speckamp, W. N.; Kaptein, B.; Boesten, W. H. J. J. Org. Chem. 1995, 60, 1733.

3) Franz, L. Z.; Uli, K. Synthesis 1999, 1785.

4) Helmboldt, H.; Hiersemann, M. Tetrahedron 2003, 59, 4031.

5) Hiersemann, M. Synthesis 2000, 1279.

6) Broggini, G.; Molteni G.; Pilati T. Tetrahedron: Asymmetry 2000, 11, 1975.

7) a) Burger, A.; Ullyot, G. E. J. Org. Chem. 1947, 12, 342. b) Troostwijk, C. B.; Kellogg, M. Chem. Comm. 1977, 346.

8) Schuetz, R. D.; Millard, F. W. J. Org. Chem. 1959, 24, 297.

9) Baldwin, J. E.; Black, K. A. J. Org. Chem. 1983, 48, 2778. 